TRANSACTIONS OF THE

AMERICAN MATHEMATICAL SOCIETY

Volume 354, Number 9, Pages 3535-3554

S 0002-9947(02)03032-5

Article electronically published on April 30, 2002

\title{
ON THE FINITE-DIMENSIONAL DYNAMICAL SYSTEMS WITH LIMITED COMPETITION
}

\author{
XING LIANG AND JIFA JIANG
}

\begin{abstract}
The asymptotic behavior of dynamical systems with limited competition is investigated. We study index theory for fixed points, permanence, global stability, convergence everywhere and coexistence. It is shown that the system has a globally asymptotically stable fixed point if every fixed point is hyperbolic and locally asymptotically stable relative to the face it belongs to. A nice result is the necessary and sufficient conditions for the system to have a globally asymptotically stable positive fixed point. It can be used to establish the sufficient conditions for the system to persist uniformly and the convergence result for all orbits. Applications are made to time-periodic ordinary differential equations and reaction-diffusion equations.
\end{abstract}

\section{INTRODUCTION}

Recently, an approach has been found to competing dynamical systems with two species. Hess and Lazer [1] did the first work in this area. Hsu, Smith and Waltman [2], Hsu, Waltman and Ellermeyer [3, Smith and Thieme [4] and references therein continued this research.

Let $X_{1}$ and $X_{2}$ be ordered Banach spaces with positive cones $X_{1}^{+}$and $X_{2}^{+}$such that $\operatorname{Int} X_{i}^{+} \neq \emptyset$ for $i=1,2$, and let the order in both of these spaces be denoted by " $\leq$ ". We define an order $\leq_{K}$ in $X_{1} \times X_{2}$ as follows:

$$
\left(x_{1}, x_{2}\right) \leq_{K}\left(y_{1}, y_{2}\right) \Leftrightarrow x_{1} \leq y_{1} \text { and } y_{2} \leq x_{2} \text {, where } K=X_{1}^{+} \times\left(-X_{2}^{+}\right) .
$$

The coordinates of a point $\left(x_{1}, x_{2}\right) \in X_{1}^{+} \times X_{2}^{+}$are viewed as representing the population density of two species in competition with each other. A competitive system is a map $T=\left(T_{1}, T_{2}\right): X_{1}^{+} \times X_{2}^{+} \rightarrow X_{1}^{+} \times X_{2}^{+}$with the properties that an increase in $x_{1}$ coupled with a decrease in $x_{2}$ results in an increase in $T_{1}$ and a decrease in $T_{2}$ and that, symmetrically, a decrease in $x_{1}$ coupled with an increase in $x_{2}$ results in a decrease in $T_{1}$ and an increase in $T_{2}$. These natural properties imply that $T$ preserves the order $\leq_{K}$, that is, $T\left(x_{1}, x_{2}\right) \leq_{K} T\left(y_{1}, y_{2}\right)$ whenever $\left(x_{1}, x_{2}\right) \leq_{K}\left(y_{1}, y_{2}\right)$.

Hess and Lazer [1] first established this abstract model, developed the theory of exclusion and coexistence, and applied it to reaction-diffusion equations with Neumann boundary conditions. Hsu, Smith and Waltman [2] took a more topological approach, relaxing the smoothness hypotheses used by Hess and Lazer but

Received by the editors May 25, 2001.

2000 Mathematics Subject Classification. Primary 34D23, 47H07; Secondary 92B05.

Key words and phrases. Map with limited competition, index of fixed points, global stability, permanence, coexistence.

Research supported by the National Natural Science Foundation of China. 
retaining a strong compactness hypothesis and the assumption that the positive cones have nonempty interior. Very recently, Smith and Thieme [4] have been able to reduce the compactness hypothesis and, in most cases, to drop the assumption that the positive cones have nonempty interior. They have derived the conditions for stable coexistence, bi-stability and competitive exclusion, and presented a complete classification of all possible outcomes in the case that there is at most one positive fixed point representing coexistence of both species. All the results in the above-mentioned papers can only be applied to systems with exactly two species.

It is obvious that a competitive system with two species can be generalized to systems with more than two species. Precisely, letting $X_{i}$ be ordered Banach spaces with positive cones $X_{i}^{+}$having nonempty interior for $i=1,2, \ldots, n(n \geq 2)$, and letting $1 \leq k<n$, we get a cone $K=X_{1}^{+} \times \ldots \times X_{k}^{+} \times\left(-X_{k+1}^{+}\right) \times \ldots \times\left(-X_{n}^{+}\right)$in the space $X=\prod_{i=1}^{n} X_{i}$. The two competing species are replaced by two competing subcommunities, which are labelled by $I=\{1, \ldots, k\}$ and $J=\{k+1, \ldots, n\}$. $T: \prod_{i=1}^{n} X_{i}^{+} \rightarrow \prod_{i=1}^{n} X_{i}^{+}$is called a system with limited competition if $T(x) \leq_{K} T(y)$ whenever $x \leq_{K} y$, where $x=\left(x_{1}, \ldots, x_{n}\right), y=\left(y_{1}, \ldots, y_{n}\right) \in \prod_{i=1}^{n} X_{i}^{+}$. If the number of species is two, then this defined system is just the competitive one described in 1]-4]. However, when the number of species is greater than two, such maps characterize those systems with the properties that "friends of friends are friends," "friends of enemies are enemies," and "enemies of enemies are friends." For systems of ordinary differential equations, such models can be described in the following form:

$$
\dot{x}_{i}=x_{i} f_{i}\left(x_{1}, x_{2}, \ldots, x_{n}, t\right), \quad x_{i} \geq 0, \quad 1 \leq i \leq n .
$$

The Jacobians with respect to $x$ of $f=\left(f_{1}, \ldots, f_{n}\right)$ have the form

$$
D_{x} f(x, t)=\left(\begin{array}{cc}
A & -B \\
-C & D
\end{array}\right),
$$

where $A$ is a $k \times k$ matrix, $B$ is a $k \times(n-k)$ matrix, $C$ is an $(n-k) \times k$ matrix, $D$ is an $(n-k) \times(n-k)$ matrix, and each off-diagonal element of $A$ and $D$ is nonnegative, and $B$ and $C$ are nonnegative matrices.

Although Takeuchi, Adachi and Tokumaru [5] and Travis and Post [6] first considered this class of systems in an ecological context, and many others continued to study them using techniques of mathematical programming, Smith [7, 8] began to investigate the asymptotic behavior of solutions of these general equations systematically in a way consistent with the spirit described above and the theory of monotone dynamical systems pioneered by Hirsch [9]-[11].

The work in [5] -8 focuses on autonomous systems. Smith 7] determined the asymptotic behavior of (1.1) in the case that (1.2) holds, and obtained sufficient conditions for all species to persist uniformly in the sense of Butler, Waltman and Freedman [12]-14]. He also sharpened this result for the Lotka-Volterra system

$$
\dot{x}_{i}=x_{i}\left(r_{i}+\sum_{j=1}^{n} a_{i j} x_{j}\right), \quad x_{i} \geq 0, \quad 1 \leq i \leq n,
$$

and proved a result on coexistence in a globally stable positive equilibrium in the case that $M=\left(a_{i j}\right)_{n \times n}$ has the form (1.2) and is stable. For the Lotka-Volterra 
system (1.3), we should mention a remarkable result of Takeuchi and Adachi [15], in which they proved that there is an equilibrium attracting all positive initial points if $M$ is stable. More general systems were considered in [16, 17], where more elegant results were obtained. We observe that (1.1) has the property

(H1) all faces of $R_{+}^{n}$ making up the boundary of $R_{+}^{n}$ are invariant for (1.1), and that if $M$ is stable then (1.3) satisfies

(H2) every fixed point (equilibrium) is hyperbolic, and

(H3) every fixed point is locally asymptotically stable relative to the face to which it belongs.

The purpose of this paper is to study the asymptotic behavior of finite-dimensional discrete-time dynamical systems $\left\{T^{n}\right\}_{n \geq 0}$ with limited competition. Under the assumptions (H1) through (H3), we shall prove that there exists a fixed point $p$ which attracts all positive initial points. Moreover, we shall show that the two subcommunities coexist in a globally stable positive fixed point if and only if

(1) there is an index subset $L \subset N=\{1,2, \cdots, n\}$ with $L \supset I=\{1,2, \cdots, k\}$ such that the face $H_{L}^{+}=\left\{x: x_{i} \geq 0\right.$ for all $i \in L$ and $x_{k}=0$ for all $\left.k \notin L\right\}$ has a positive fixed point $u$ satisfying $\left.\frac{\partial T_{j}}{\partial x_{j}}\right|_{u}>1$ for any $j \notin L$, and

(2) there is an index subset $P \supset J=\{k+1, k+2, \cdots, n\}$ such that the face $H_{P}^{+}$ has a positive fixed point $v$ satisfying $\left.\frac{\partial T_{i}}{\partial x_{i}}\right|_{v}>1$ for any $i \notin P$.

These results unify all individual results in this direction under the most succinct conditions. This shows that conditions (1) and (2) are the essence of coexisting in a globally stable positive fixed point, and nearly gives necessary and sufficient conditions for systems with limited competition to coexist in a globally stable positive fixed point. Furthermore, assuming (H1), (1) and (2) hold, we conclude that there is an attractor block lying in the interior of the positive octant with fixed points at two corners, each of which attracts an unbounded open set of positive initial points. By applying Terešćák's result in [18], almost all orbits are asymptotic to periodic points if $T$ is strongly order-preserving in the interior of $R_{+}^{n}$. The convergent result for all orbits is provided under suitable conditions. We also apply our abstract results to periodic ordinary differential equations and reaction-diffusion Lotka-Volterra systems with Neumann boundary conditions:

$$
\left\{\begin{array}{l}
\frac{\partial u}{\partial t}=\operatorname{diag}\left(d_{1}, \cdots, d_{n}\right) \Delta u+\operatorname{diag}(u)(r+M u) \text { in } \Omega_{T} \\
\frac{\partial u}{\partial \nu}=0 \text { on } \partial \Omega_{T} \\
u(x, 0)=u^{0}(x) \text { in } \Omega
\end{array}\right.
$$

where $M$ has the form (1.2). As far as we know, most work on (1.4) focusses on two species, and the best results so far were obtained in [19]-[21], where the matrix $M=\left(a_{i j}\right)_{2 \times 2}$ and the corresponding two-dimensional system (1.3) are assumed to satisfy the hypotheses (H1) through (H3) in all three cases. They proved that there is a steady state attracting all positive initial value functions. Applying our abstract results, we can generalize the best results for two species to any species. Precisely, if $M=\left(a_{i j}\right)_{n \times n}$ has the form (1.2) and (1.3) satisfies (H1) through (H3), then there is a steady state attracting all $u^{0}(x) \geq 0$ with $u_{i}^{0}(x) \not \equiv 0$ for all $i$. The necessary and sufficient conditions for (1.4) to coexist in a positive steady state or to exclude in a boundary steady state are also provided. 


\section{Preliminary}

In this section, we will introduce some notation, establish some conventions, and describe some results which are essential tools in the later sections.

Let $R_{+}^{n}=\left\{x \in R^{n}: x_{i} \geq 0\right.$ for $\left.1 \leq i \leq n\right\}$ denote the nonnegative octant, and let $\operatorname{Int} R_{+}^{n}=\left\{x \in R^{n}: x_{i}>0\right.$ for $\left.1 \leq i \leq n\right\}$ denote the interior of $R_{+}^{n}$. Define the set $K=\left\{x \in R^{n}: x_{i} \geq 0\right.$ for $1 \leq i \leq k$ and $x_{j} \leq 0$ for $\left.k+1 \leq j \leq n\right\}$. We know both $R_{+}^{n}$ and $K$ are cones in $R^{n}$. We write $x \leq y\left(x \leq_{K} y\right)$ and $y \geq x\left(y \geq_{K} x\right)$ whenever $y-x \in R_{+}^{n}(y-x \in K), x<y\left(x<_{K} y\right)$ and $y>x\left(y>_{K} x\right)$ whenever $x \leq y\left(x \leq_{K} y\right)$ and $x \neq y$. If $x, y \in R^{n}$ and $x \leq y\left(x \leq_{K} y\right)$, we let $[x, y]=\left\{z \in R^{n}: x \leq z \leq y\right\}\left([x, y]_{K}=\left\{z \in R^{n}: x \leq_{K} z \leq_{K} y\right\}\right)$.

Suppose that $A$ is an $n \times m$ matrix. Then we write $A \geq 0$ if $a_{i j} \geq 0$ for all $i$ and $j$. If $M$ is an $n \times n$ matrix, and

$$
M=\left(\begin{array}{cc}
A & -B \\
-C & D
\end{array}\right)
$$

where $A$ is a $k \times k$ matrix, $B$ is a $k \times(n-k)$ matrix, $C$ is an $(n-k) \times k$ matrix, $D$ is an $(n-k) \times(n-k)$ matrix and $A, B, C, D \geq 0$, then we write $M \geq_{K} 0$. It is easy to see that $M(K) \subset K$.

We will reserve the letter $n$ for the dimension of $R^{n}$, and $N=\{1,2, \cdots, n\}$. Let $L$ be a subset of $N$ and $\bar{L}=N \backslash L$ be its complementary set in $N$. We define the sets $H_{L}^{+}=\left\{x \in R_{+}^{n}: x_{j}=0\right.$ for $\left.j \in \bar{L}\right\}$ and $\operatorname{Int} H_{L}^{+}=\left\{x \in H_{L}^{+}: x_{i}>0\right.$ for all $i \in L\}$. In particular, if $L=\emptyset$, then $H_{L}^{+}=\operatorname{Int} H_{L}^{+}=\{(0,0, \ldots, 0)\}$.

A continuous map $S: R_{+}^{n} \rightarrow R_{+}^{n}$ is said to be cooperative if $S(x)<S(y)$ whenever $x<y$ with $x, y \in R_{+}^{n}$, and $T: R_{+}^{n} \rightarrow R_{+}^{n}$ is said to be a map with limited competition if $T(x)<_{K} T(y)$ whenever $x<_{K} y$ with $x, y \in R_{+}^{n}$. In particular, if $k=0$, then $T$ is also cooperative. We note that a map with limited competition is often said to be type- $K$ monotone (see [7], [16]). In this paper we always assume that $S, T \in C^{1}\left(R_{+}^{n}, R_{+}^{n}\right)$ and $S$ is a cooperative map, and $T$ is a map with limited competition. We also always assume that each orbit of $S$ or $T$ is bounded. We denote by $S_{1}, S_{2}, \ldots, S_{n}$ and $T_{1}, T_{2}, \ldots, T_{n}$ the components of $S$ and $T$ respectively. It is easy to see that $D S=\left(\frac{\partial S_{i}}{\partial x_{j}}\right)_{n \times n} \geq 0$ and $D T=\left(\frac{\partial T_{i}}{\partial x_{j}}\right)_{n \times n} \geq_{K} 0$.

Now, we give the main hypotheses on $T$.

(AT) $T\left(\operatorname{Int} H_{L}^{+}\right) \subset \operatorname{Int} H_{L}^{+}$for any $L \subset N$.

(BT) If $x_{0}$ is a fixed point of $T$ with $x_{0} \in \operatorname{Int} H_{L}^{+}$, then $x_{0}$ is locally asymptotically stable with respect to $H_{L}^{+}$.

(CT) Each fixed point $x_{0}$ of $T$ is hyperbolic.

For the cooperative map $S$, we still suppose that the same hypotheses hold, but use (AS), (BS) and (CS) to replace (AT), (BT) and (CT) respectively.

If a map $T$ satisfies (AT) and a point $x \in H_{L}^{+}$, then for any $i \in \bar{L}, j \neq i$, $\left.\frac{\partial T_{i}}{\partial x_{j}}\right|_{x}=0$ and $\left.\frac{\partial T_{i}}{\partial x_{i}}\right|_{x}$ is an eigenvalue of $D T(x)$. A cooperative map $S$ has the similar property if it satisfies (AS).

Suppose $I=\{1,2, \ldots, k\}$ and $J=\{k+1, k+2, \ldots, n\}$. Then the restriction of $T$ to $H_{I}^{+}$or $H_{J}^{+}$is cooperative. If $T$ satisfies (AT), (BT) and (CT), then the restriction of $T$ to $H_{I}^{+}$or $H_{J}^{+}$satisfies (AS), (BS), and (CS).

In sections 3 through 6 we use $\omega(x)$ to denote the omega limit set of $x$ under $S$ or $T$; if $\omega(x)=\{y\}$ has only one element, then we write $\omega(x)=y$. 


\section{Global Stability FOR COOPERATIVE Systems}

The object of this section is to prove the following theorem.

Theorem 3.1. Suppose that $S: R_{+}^{n} \rightarrow R_{+}^{n}$ satisfies the hypotheses (AS), (BS) and (CS). Then there is a fixed point $p \in R_{+}^{n}$ such that $\omega(x)=p$ for any $x \in$ Int $R_{+}^{n}$. Furthermore, a necessary and sufficient condition for $S$ to have a globally asymptotically stable positive fixed point is that there exist a subset $L \subset N$ and a boundary fixed point $c \in \operatorname{Int} H_{L}^{+}$such that $\left.\frac{\partial T_{i}}{\partial x_{i}}\right|_{c}>1$ for all $i \in \bar{L}$.

To prove this theorem, we need the following lemma.

Lemma 3.2. Suppose that $S: R_{+}^{n} \rightarrow R_{+}^{n}$ satisfies the hypotheses (AS), (BS) and (CS), and that there exists a locally asymptotically stable fixed point $p$ in $R_{+}^{n}$. Then $\omega(x)=p$ for any $x \in \operatorname{Int} R_{+}^{n}$. Furthermore, let $L=\left\{i: p_{i}>0\right\}$. Then the domain of attraction of $p$ is $\Omega=\left\{x \in R_{+}^{n}: x_{i}>0\right.$ for all $\left.i \in L\right\}$.

Proof. Since (AS) implies that $H_{P}^{+}$is invariant under $S$ for any $P \subset N$, we shall prove this lemma by induction on the dimension $n$ of $R_{+}^{n}$. The result is obvious if $n=1$. So assume for induction that the lemma is true when the dimension of the system is less than $n$. Now, we consider an $n$-dimensional system. First, because $p$ is locally asymptotically stable, there is no other fixed point in $\Omega_{1}=p+R_{+}^{n}$. In fact, if there is another fixed point in $\Omega_{1}$, then we can find a fixed point $\bar{p}$ in $\Omega_{1}$ such that there is no other fixed point distinct from $p$ and $\bar{p}$ in $[p, \bar{p}]$, because $p$ is locally asymptotically stable and there is no other fixed point in a small neighborhood of p. Assume that $\bar{p} \in \operatorname{Int} H_{L_{1}}^{+}$for some $L_{1} \subset N$. Then $[p, \bar{p}] \subset H_{L_{1}}^{+}$. By (BS), $\bar{p}$ is locally asymptotically stable with respect to $H_{L_{1}}^{+}$. In particular, $p$ and $\bar{p}$ are locally asymptotically stable with respect to $[p, \bar{p}]$. By the result in Dancer and Hess [22], $S$ has a fixed point distinct from $p$ and $\bar{p}$ in $[p, \bar{p}]$, contradicting the conclusion that we have just shown. Because $\Omega_{1}$ is an invariant set of $S, p$ attracts all points in $\Omega_{1}$ by the result in Jiang [23]. In the following we divide the proof into two cases.

Case (1): $p \notin \operatorname{Int} R_{+}^{n}$. Obviously, there is some $L_{2} \neq N$ such that $p \in \operatorname{Int} H_{L_{2}}^{+}$. The induction assumption implies that the domain of attraction of $p$ includes the set $\Omega_{2}=\left\{x \in R_{+}^{n}: 0<x_{i} \leq p_{i}\right.$ for $i \in L_{2}$ and $x_{j}=0$ for $\left.j \in \bar{L}_{2}\right\}$. For any $x \in \Omega$, we can find $x^{1} \in \Omega_{1}$ and $x^{2} \in \Omega_{2}$ such that $x^{2} \leq x \leq x^{1}$. Because of the monotonicity of $S, \omega\left(x^{2}\right)=\omega(x)=\omega\left(x^{1}\right)=p$.

Case (2): $p \in \operatorname{Int} R_{+}^{n}$. Let $N_{i}=N \backslash\{i\}$. Denote $x-x_{i} e_{i}$ by $x_{N_{i}}$ for any $x \in$ $R_{+}^{n}$, where $x=\left(x_{1}, x_{2}, \cdots, x_{i}, \cdots, x_{n}\right)$ and $e_{i}=\left(0,0, \cdots, 1_{i}, 0, \cdots, 0\right)$. Obviously, $x_{N_{i}} \in \operatorname{Int} H_{N_{i}}^{+}$for any $x \in \operatorname{Int} R_{+}^{n}$. Since $p_{N_{i}}<p, S\left(p_{N_{i}}\right)<S(p)=p$, i.e., $S_{j}\left(p_{N_{i}}\right) \leq$ $p_{j}$ for $j \in N_{i}$. Hence, $S\left(p_{N_{i}}\right) \leq p_{N_{i}}$. It is easy to see that there exists a fixed point $q_{N_{i}}$ such that $\omega\left(p_{N_{i}}\right)=q_{N_{i}}$. The hypothesis (CS) shows that the local stable set of $q_{N_{i}}$ is a manifold (with boundary). By (BS), there is $\widetilde{N}_{i} \subset N_{i}$ such that $q_{N_{i}} \in \operatorname{Int} H_{\widetilde{N}_{i}}^{+}$and $q_{N_{i}}$ is locally asymptotically stable with respect to $H_{\widetilde{N}_{i}}^{+}$. On the other hand, $q_{N_{i}}$ attracts all points in $\left[q_{N_{i}}, p_{N_{i}}\right]$. So the local stable manifold (with boundary) of $q_{N_{i}}$ includes a neighborhood of $q_{N_{i}}$ in $H_{N_{i}}^{+}$. The induction assumption shows that the domain of attraction of $q_{N_{i}}$ includes $\operatorname{Int} H_{N_{i}}^{+}$.

Define

$$
q=\left(q_{1}, q_{2}, \ldots, q_{n}\right) \text { with } q_{i}=\max _{j \in N}\left(q_{N_{j}}\right)_{i}
$$


Then $q_{N_{i}} \leq q$ for any $i \in N$, and if $q_{N_{i}} \leq x$ for any $i \in N$, then $q \leq x$. On the other hand, $q_{N_{i}}=S\left(q_{N_{i}}\right) \leq S(q)$, which implies $q \leq S(q)$. So there exists a fixed point $w$ such that $\omega(q)=w \geq q$.

For any $x \in \operatorname{Int} R_{+}^{n}, \omega(x) \geq \omega\left(x_{N_{i}}\right)=q_{N_{i}}$ for any $i \in N$. So $\omega(x) \geq q$; in particular, $\omega(p)=p \geq q$. If $q \in \operatorname{Int} R_{+}^{n}$, then $\omega(q)=p$. In fact, $q \leq w=\omega(q) \leq p$. Since $w \in \operatorname{Int} R_{+}^{n}$, the result just proved in the first paragraph shows that there is no other fixed point in $w+R_{+}^{n}$, and so $w=p$. Because $\omega(x) \geq q$ for any $x \in \operatorname{Int} R_{+}^{n}$, we have $\omega(y) \geq \omega(q)=p$ for any $y \in \omega(x)$. Furthermore, $\omega(y)=p$, since $\Omega_{1}$ is contained in the attracting domain of $p$. So $\{p\}=\omega(y) \subset \omega(x)$. However, $p$ is locally asymptotically stable; hence $\omega(x)=p$.

If $q \notin$ Int $R_{+}^{n}$, then there is some $i \in N$ such that $q \in H_{N_{i}}^{+}$. Since $q \geq q_{N_{i}}$ and $q_{N_{i}}$ attracts all points in $\operatorname{Int} H_{N_{i}}^{+}$by the induction assumption, $\omega(q)=w=q_{N_{i}}$. Because $\omega(q) \geq q \geq q_{N_{i}}$, we get $q=q_{N_{i}}$. Since $q$ is locally asymptotically stable with respect to $H_{N_{i}}^{+}$, we must have $\left.\frac{\partial S_{i}}{\partial x_{i}}\right|_{q}>1$. Otherwise, $q$ is locally asymptotically stable in $R_{+}^{n}$. Based on the result in case (1), $q$ attracts all points in Int $R_{+}^{n}$, contradicting the fact that $p \in \operatorname{Int} R_{+}^{n}$ is a fixed point.

Now, we show that $q=q_{N_{i}} \in \operatorname{Int} H_{N_{i}}^{+}$. Otherwise, there is some $j \in N_{i}$ such that $\left(q_{N_{i}}\right)_{j}=0$. Then $q_{N_{i}}^{\delta}=q_{N_{i}}+\delta e_{i} \in H_{N_{j}}^{+}$for any positive number $\delta$. Because $\left.\frac{\partial S_{i}}{\partial x_{i}}\right|_{q}>1$ and $\left.\frac{\partial S_{i}}{\partial x_{j}}\right|_{q}=0, j \neq i$, we get

$$
\begin{aligned}
S_{i}\left(q_{N_{i}}^{\delta}\right) & =S_{i}\left(q_{N_{i}}^{\delta}\right)-S_{i}\left(q_{N_{i}}\right) \\
& =\left(\int_{0}^{1} D S\left(s q_{N_{i}}^{\delta}+(1-s) q_{N_{i}}\right) d s\left(q_{N_{i}}^{\delta}-q_{N_{i}}\right)\right)_{i} \\
& \geq \delta=\left(q_{N_{i}}^{\delta}-q\right)_{i}
\end{aligned}
$$

for sufficiently small $\delta$. This implies that $S\left(q_{N_{i}}^{\delta}\right) \geq q_{N_{i}}^{\delta}>q$. Thus, there is a fixed point $\bar{w}$ such that $\omega\left(q_{N_{i}}^{\delta}\right)=\bar{w}$ with $\bar{w}>q$. By $q_{N_{i}}^{\delta} \in H_{N_{j}}^{+}$and (AS), $S\left(q_{N_{i}}^{\delta}\right) \in H_{N_{j}}^{+}$ and hence $\bar{w} \in H_{N_{j}}^{+}$, and $\bar{w}=\omega(\bar{w})>q \geq q_{N_{j}}$. On the other hand, since $\bar{w} \in H_{N_{j}}^{+}$ and $\bar{w} \geq q_{N_{j}}$, we have $\omega(\bar{w})=q_{N_{j}}$, a contradiction.

By the proof of the previous paragraphs, we know that $q=q_{N_{i}} \in \operatorname{Int} H_{N_{i}}^{+}$and $\omega\left(q^{\delta}\right) \in \operatorname{Int} R_{+}^{n}$ is a fixed point with $q^{\delta}=q+\delta e_{i}$ for $0<\delta<\delta_{0}$. Furthermore, $\omega\left(q^{\delta}\right) \leq p$. But since $\omega\left(q^{\delta}\right) \in \operatorname{Int} R_{+}^{n}$ is asymptotically stable, there is no other fixed point in $\omega\left(q^{\delta}\right)+R_{+}^{n}$. So $\omega\left(q^{\delta}\right)=p$. By the induction assumption, $q$ attracts Int $H_{N_{i}}^{+}$. It follows from the hyperbolicity and $\left.\frac{\partial S_{i}}{\partial x_{i}}\right|_{q}>1$ that the dimension of the stable manifold $W^{s}(q)$ of $q$ is $n-1$. Hence $W^{s}(q)=\operatorname{Int} H_{N_{i}}^{+}$. This proves there cannot exist an $x \in \operatorname{Int} R_{+}^{n}$ such that $\omega(x)=q$. We claim that $\omega(x)=p$ for all $x \in \operatorname{Int} R_{+}^{n}$. Suppose not. Then there exists an $x^{0} \in \operatorname{Int} R_{+}^{n}$ such that $\omega\left(x^{0}\right) \neq p$. This implies that there is a point $y \in \omega\left(x^{0}\right)$ with $y \neq q, p$. It is easy to see that $\omega(x) \subset[q, p]$ for all $x \in \operatorname{Int} R_{+}^{n}$. Therefore, $q<y<p$. Choose $\delta \in\left(0, \delta_{0}\right)$ such that $q^{\delta}<y<p$. It follows from $\omega\left(q^{\delta}\right)=p$ that $\omega(y)=p$, i.e., $p \in \omega(y) \subset \omega\left(x^{0}\right)$, a contradiction. This completes the proof.

Proof of Theorem 3.1. The theorem is true when $n=1$. Now we proceed by induction on the dimension $n$ of $R_{+}^{n}$. Suppose that the theorem is true when the dimension of the systems is less than $n$. Then we consider an $n$-dimensional system 
and look for a fixed point $p$ such that it attracts the set $\Omega=\left\{x \in R_{+}^{n}: x_{i}>\right.$ 0 for every $i$ with $\left.p_{i}>0\right\}$. By the induction assumption, for any $i \in N$, there is a fixed point $q_{N_{i}} \in H_{N_{i}}^{+}$with $q_{N_{i}} \in \operatorname{Int} H_{L}^{+}$for some $L \subset N_{i}$ such that $q_{N_{i}}$ attracts all points in $\left\{x \in H_{N_{i}}^{+}: x_{i}>0\right.$ for $\left.i \in L\right\}$. Suppose that $q$ is the point defined by (3.1). If $q \in \operatorname{Int} R_{+}^{n}$, then $S(q) \geq q$ by the proof of Lemma 3.2. So $\omega(q) \in \operatorname{Int} R_{+}^{n}$ is a fixed point, and we denote it by $p$. It follows from (BS) that $p$ is locally asymptotically stable in Int $R_{+}^{n}$. By Lemma 3.2, Int $R_{+}^{n}$ is the domain of attraction of $p$.

If $q \notin \operatorname{Int} R_{+}^{n}$, then the proof of Lemma 3.2 shows that there is an $i \in N$ such that $q=q_{N_{i}}$ attracts Int $H_{N_{i}}^{+}$. If $q$ is locally asymptotically stable, then, applying Lemma 3.2 to $q$, we obtain that $q$ attracts the set $\Omega=\left\{x \in R_{+}^{n}: x_{j}>0\right.$ for each $j$ with $q_{j}>$ $0\}$. Therefore, $q$ is the desired fixed point. Otherwise $\left.\frac{\partial S_{i}}{\partial x_{i}}\right|_{q_{N_{i}}}>1$, and hence, by the proof of Lemma 3.2, $q_{N_{i}} \in \operatorname{Int} H_{N_{i}}^{+}$, and $q_{N_{i}}^{\delta}=q_{N_{i}}+\delta e_{i} \in \operatorname{Int} R_{+}^{n}, S\left(q_{N_{i}}^{\delta}\right) \geq q_{N_{i}}^{\delta}$. Hence $\omega\left(q_{N_{i}}^{\delta}\right) \in \operatorname{Int} R_{+}^{n}$ is a fixed point. If we denote the positive fixed point $\omega\left(q_{N_{i}}^{\delta}\right)$ by $p$, then $p$ is locally asymptotically stable by (BS). Lemma 3.2 implies $p$ attracts all points in Int $R_{+}^{n}$. Such a fixed point $p$ is a desired one.

In the following, we shall prove the remaining part of the theorem. Suppose that $S$ has a globally asymptotically stable positive fixed point $p$. Then the fixed point 0 is not asymptotically stable. Indeed, if 0 is asymptotically stable, then 0 is globally asymptotically stable by Lemma 3.2, a contradiction. Without loss of generality, suppose $\left.\frac{\partial S_{1}}{\partial x_{1}}\right|_{0}>1$. Then, it is easy to see that $S\left(\epsilon e_{1}\right)>\epsilon e_{1}$ for all sufficiently small positive numbers $\epsilon$. So $a=\omega\left(\epsilon e_{1}\right) \in \operatorname{Int} H_{\{1\}}^{+}$is a fixed point with $0<a<p$. Similarly, $a$ is not asymptotically stable. Suppose $\left.\frac{\partial S_{2}}{\partial x_{2}}\right|_{a}>1$. Then $S\left(a+\epsilon e_{2}\right)>a+\epsilon e_{2}$ for all sufficiently small positive numbers $\epsilon$. There is a fixed point $b=\omega\left(a+\epsilon e_{2}\right) \in \operatorname{Int} H_{\{1,2\}}^{+}$with $a<b<p$ that is not asymptotically stable. In this way, we can find a fixed point $c \in \operatorname{Int} H_{N_{i}}^{+}$such that $c$ is not asymptotically stable. This means $\left.\frac{\partial S_{i}}{\partial x_{i}}\right|_{c}>1$. The proof of necessity is complete.

On the other hand, suppose there exist a subset $L \subset N$ and a boundary fixed point $c \in \operatorname{Int} H_{L}^{+}$such that $\left.\frac{\partial S_{i}}{\partial x_{i}}\right|_{c}>0$ for all $i \in \bar{L}$. Without loss of generality, suppose $L=\{1, \cdots, l\}, c=\left(c_{1}, \cdots, c_{l}, 0, \cdots, 0\right)$ and $\bar{c}=\left(c_{1}, \cdots, c_{l}, \epsilon, \cdots, \epsilon\right)$, where $\epsilon$ is a sufficiently small positive number. Then $\bar{c} \in \operatorname{Int} R_{+}^{n}$ and $S(\bar{c})>\bar{c}$. Therefore, $p=\omega(\bar{c}) \in \operatorname{Int} R_{+}^{n}$ is a positive fixed point that is asymptotically stable by (BS). Furthermore, it follows from Lemma 3.2 that $p$ is globally asymptotically stable. The proof is complete.

Remark 3.3. For autonomous ordinary differential equations, similar results were proved in [25, 26]. Theorem 3.1 can be viewed as a unified generalization of such individual results.

\section{Global Stability For Systems with Limited COMPetition}

The object of this section is to prove the following theorem, which is about the global stability of systems with limited competition.

Theorem 4.1. Suppose that $T: R_{+}^{n} \rightarrow R_{+}^{n}$ satisfies the hypotheses (AT), (BT) and $(\mathrm{CT})$. Then there is a fixed point $p \in R_{+}^{n}$ such that $\omega(x)=p$ for any $x \in$ Int $R_{+}^{n}$. Furthermore, let $L=\left\{i: p_{i}>0\right\}$. Then the domain of attraction of $p$ is $\Omega=\left\{x \in R_{+}^{n}: x_{i}>0\right.$ for all $\left.i \in L\right\}$. 
To prove this theorem, we first give a lemma about the index of fixed points.

Lemma 4.2. Suppose that $T: R_{+}^{n} \rightarrow R_{+}^{n}$ satisfies the hypotheses (AT), (BT) and (CT). Let $U=R_{+}^{n}$ or $U=H_{L}^{+}$or $U=[p, q]_{K}$, where $p$ and $q$ are fixed points of $T$. Then for any fixed point $x^{0} \in U$, either $i\left(T, U, x^{0}\right)=0$ or $i\left(T, U, x^{0}\right)=1$. Furthermore, $i\left(T, U, x^{0}\right)=\left.0 \Leftrightarrow D T\right|_{x^{0}}$ has at least one eigenvalue with modulus larger than 1.

Proof. Obviously, $U$ is an invariant set under $T$. If there exists $L \neq N$ such that $U \subset H_{L}^{+}$, then the restriction of $T$ to $H_{L}^{+}$also satisfies (AT), (BT) and (CT). So we only need to consider the case that $U \cap \operatorname{Int} R_{+}^{n} \neq \emptyset$.

Suppose that $x^{0} \in U$ is a fixed point. Then

$$
D T\left(x^{0}\right)\left(x-x^{0}\right)=\lim _{\tau \rightarrow 0^{+}} \tau^{-1}\left(T\left(\tau\left(x-x^{0}\right)+x^{0}\right)-T\left(x^{0}\right)\right) \text { for any } x \in U .
$$

Let $P=x^{0}+\bigcup_{t=0}^{+\infty} t\left(U-x^{0}\right)$. Then it is easy to see that $P$ is a closed set. For any $x \in U$ and $0 \leq \tau \leq 1, \tau\left(x-x^{0}\right)+x^{0} \in U$. Hence $T\left(\tau\left(x-x^{0}\right)+x^{0}\right) \in U$. So $\tau^{-1}\left(T\left(\tau\left(x-x^{0}\right)+x^{0}\right)-T\left(x^{0}\right)\right)+x^{0} \in P$. This implies that $D T\left(x^{0}\right)\left(x-x^{0}\right)+x^{0} \in P$.

Suppose that $B\left(x^{0}, \delta\right)$ is a closed ball in $R_{+}^{n}$ whose center is $x^{0}$ and radius is $\delta$, where $\delta$ is sufficiently small so that $B^{+}\left(x^{0}, \delta\right)=B\left(x^{0}, \delta\right) \cap P \subset U$. Because $D T\left(x^{0}\right)$ is a linear map, there is an $\epsilon$ such that $D T\left(x^{0}\right)\left(x-x^{0}\right)+x^{0} \in B^{+}\left(x^{0}, \delta\right) \subset U$ for any $x \in B^{+}\left(x^{0}, \epsilon\right)$.

Since $D T\left(x^{0}\right)$ is hyperbolic, there is $\alpha>0$ such that

$$
\left\|x-\left(x^{0}+D T\left(x^{0}\right)\left(x-x^{0}\right)\right)\right\| \geq \alpha\left\|x-x^{0}\right\| .
$$

Choose $\sigma<\epsilon$ such that $\left\|T(x)-T\left(x^{0}\right)-D T\left(x^{0}\right)\left(x-x^{0}\right)\right\| \leq \frac{\alpha}{2}\left\|x-x^{0}\right\|$ for any $x \in B^{+}\left(x^{0}, \sigma\right)$. For any $y$ with $\|y\|<\sigma \alpha / 2$ and $x^{0}+y \in U$, and any $x \in U$ with $\left\|x-x^{0}\right\|=\sigma$,

$$
\begin{aligned}
& \left\|x-(1-\lambda)\left(D T\left(x^{0}\right)\left(x-x^{0}\right)+x^{0}+y\right)-\lambda T(x)\right\| \\
= & \left\|\left(x-D T\left(x^{0}\right)\left(x-x^{0}\right)-x^{0}\right)+\lambda\left(D T\left(x^{0}\right)\left(x-x^{0}\right)+x^{0}-T(x)\right)-(1-\lambda) y\right\| \\
\geq & \left\|x-D T\left(x^{0}\right)\left(x-x^{0}\right)-x^{0}\right\|-\lambda\left\|D T\left(x^{0}\right)\left(x-x^{0}\right)+x^{0}-T(x)\right\|-(1-\lambda)\|y\| \\
> & \alpha \sigma-\alpha \sigma / 2-\alpha \sigma / 2=0 .
\end{aligned}
$$

For any $\lambda \in[0,1]$, the homotopy invariance of the fixed point index (e.g., [24] implies $i\left(T, U, x^{0}\right)=i\left(D T\left(x^{0}\right)\left(x-x^{0}\right)+x^{0}+y, U, x^{0}\right)$.

(1) Assume that $D T\left(x^{0}\right)$ has no eigenvalue whose modulus is larger than 1 . Then choose $y=0$. The homotopy invariance implies

$$
\begin{aligned}
& i\left(T, U, x^{0}\right) \\
= & i\left(D T\left(x^{0}\right)\left(x-x^{0}\right)+x^{0}, U, x^{0}\right) \\
= & i\left(\lambda D T\left(x^{0}\right)\left(x-x^{0}\right)+x^{0}, U, x^{0}\right) \quad \lambda \in[0,1] \\
= & i\left(x^{0}, U, x^{0}\right)=1 .
\end{aligned}
$$

(2) $D T\left(x^{0}\right)$ has an eigenvalue whose modulus is larger than 1 . The hypothesis (BT) implies $x^{0} \notin \operatorname{Int} R_{+}^{n}$; hence there is $L \neq N$ such that $x^{0} \in \operatorname{Int} H_{L}^{+}$. Without loss of generality, suppose $x^{0}=\left(x_{L}^{0}, 0\right)$, where $x_{L}^{0} \in \operatorname{Int} R_{+}^{l}$ with $l=\# L$. Then $D T\left(x^{0}\right)$ has the following form:

$$
M=\left(\begin{array}{cc}
A & B \\
0 & D
\end{array}\right)
$$


where $A$ is an $l \times l$ matrix, all of whose eigenvalues have moduli less than 1 , and $D=\left.\operatorname{diag}\left(\frac{\partial T_{j}}{\partial x_{j}}\right)\right|_{x^{0}}, j \in \bar{L}$. It is easy to see if $\lambda$ is one eigenvalue of $D T\left(x^{0}\right)$ whose modulus is larger than 1 , then there is some $i \in \bar{L}$ such that $\lambda=\left.\frac{\partial T_{i}}{\partial x_{i}}\right|_{x^{0}}$.

On the other hand, $U=[p, q]_{K}$ can be rewritten as the form $\left[\left(p_{I}, q_{J}\right),\left(q_{I}, p_{J}\right)\right]$, where $\left(p_{I}, q_{J}\right)$ and $\left(q_{I}, p_{J}\right)$ are elements of $R_{+}^{n}, p-\left(p_{I}, q_{J}\right) \in H_{J}^{+}, q-\left(p_{I}, q_{J}\right) \in$ $H_{I}^{+},\left(q_{I}, p_{J}\right)-p \in H_{I}^{+},\left(q_{I}, p_{J}\right)-q \in H_{J}^{+}$. Because $U \cap \operatorname{Int} R_{+}^{n} \neq \emptyset,\left(q_{I}, p_{J}\right) \in \operatorname{Int} R_{+}^{n}$. Since $x^{0} \in U$ and $\left(q_{I}, p_{J}\right) \in \operatorname{Int} R_{+}^{n}$, there is a sufficiently small $a$ such that $x^{0}+a e_{i} \in$ $U$ for $i \in \bar{L}$. We shall show that $x-\left(D T\left(x^{0}\right)\left(x-x^{0}\right)+x^{0}\right)=\beta e_{i}$ has no solution in $B^{+}\left(x^{0}, \sigma\right)$ for any $\beta>0$. In fact, $x_{i}^{0}=0, x_{i} \geq 0$. Let $A_{i}$ be the $i$-th component of $x-\left(D T\left(x^{0}\right)\left(x-x^{0}\right)+x^{0}\right)$. Then

$$
A_{i}=\left(1-\partial T_{i} /\left.\partial x_{i}\right|_{x^{0}}\right)\left(x_{i}-x_{i}^{0}\right)=(1-\lambda)\left(x_{i}-x_{i}^{0}\right) \leq 0,
$$

but the $i$-th component of $\beta e_{i}$ is larger than 0 .

Let $y=\beta e_{i}$, where $\beta$ is sufficiently small so that $\|y\|<\sigma \alpha / 2$. Then

$$
i\left(T, U, x^{0}\right)=i\left(D T\left(x^{0}\right)\left(x-x^{0}\right)+x^{0}+y, U, x^{0}\right) .
$$

By the above proof, $D T\left(x^{0}\right)\left(x-x^{0}\right)+x^{0}+y$ has no fixed point in $B^{+}\left(x^{0}, \sigma\right)$, which implies $i\left(D T\left(x^{0}\right)\left(x-x^{0}\right)+x^{0}+y, U, x^{0}\right)=0$. So $i\left(T, U, x^{0}\right)=0$.

In the following, we will show if there exists a fixed point that is locally asymptotically stable, then it is globally asymptotically stable.

Lemma 4.3. Suppose that $T: R_{+}^{n} \rightarrow R_{+}^{n}$ satisfies the hypotheses (AT), (BT) and (CT). Assume that there exists a fixed point a such that it attracts $\operatorname{Int} H_{L}^{+}$with $L \supset J, a_{i}>0$ for $i \in L \cap I$, and $\left.\frac{\partial T_{1}}{\partial x_{l}}\right|_{a}>1$ for some $l \in I \backslash L$. Then there is a fixed point $b \in H_{L_{1}}^{+}$with $L_{1}=L \cup\{l\}$ such that

(i) $b_{i}>0$ for any $i \in I \cap L_{1}$,

(ii) $b$ attracts the set

$$
\begin{gathered}
\Gamma=\left\{x \in H_{L_{1}}^{+}: 0<x_{i}<b_{i} \text { for } i \in I \cap L_{1}, x_{i^{\prime}}=0 \text { for } i^{\prime} \in I \backslash L_{1},\right. \\
\text { and } \left.x_{j}>b_{j} \text { for any } j \in J\right\},
\end{gathered}
$$

(iii) if the fixed point $p \geq_{K}$ a with $p_{l}>0$, then $b \leq_{K} p$.

Furthermore, the same conclusion holds if $I$ and $J$ are switched.

Proof. To be specific, we assume $L=\{m, m+1, \cdots, n\}$ with $m \leq k+1$. Since $a$ attracts Int $H_{L}^{+}$, we have $a=\left(0, \cdots, 0, a_{m}, a_{m+1}, \cdots, a_{n}\right)$ with $a_{i}>0$ for $m \leq i \leq k$. By $\left.\frac{\partial T_{l}}{\partial x_{l}}\right|_{a}>1$, we have $l<m$ and $T_{l}\left(a+\delta e_{l}\right)>\delta$ for $0<\delta \ll 1$. Let $\bar{a}=a+\delta e_{l}$. Then the monotonicity implies that $T(\bar{a})>_{K} \bar{a}$. Hence $\omega(\bar{a})=b>_{K} \bar{a}$. It is easy to see that $b$ satisfies (i) and is independent of $\delta$ for $0<\delta \ll 1$.

Set $x \in \Gamma$. Then $x-x_{l} e_{l}<_{K} x<_{K} b$. Obviously, $x-x_{l} e_{l} \in \operatorname{Int} H_{L}^{+}$. Therefore, $a=\omega\left(x-x_{l} e_{l}\right) \leq_{K} \omega(x) \leq_{K} b$ by the assumption and monotonicity, that is, $\omega(x) \subset[a, b]_{K}$ for any $x \in \Gamma$.

Because $T$ is continuously differentiable and $\left.\frac{\partial T_{l}}{\partial x_{l}}\right|_{a}>1$, there is a point $\widehat{a}=$ $\left(0, \ldots, 0, \widehat{a}_{m}, \ldots, \widehat{a}_{k}, \ldots, \widehat{a}_{n}\right)$ with $0<\widehat{a}_{i}<a_{i}$ for $m \leq i \leq k$ and $\widehat{a}_{j}>a_{j}$ for any $j \in J$ such that $\left.\frac{\partial T_{l}}{\partial x_{l}}\right|_{\widehat{a}}>1$, which implies that there is a $\delta_{0}>0$ such that $T_{l}\left(\widehat{a}+\epsilon e_{l}\right) \geq \epsilon$ for $0 \leq \epsilon \leq \delta_{0}$.

From now on we fix $x \in \Gamma$. From $\omega(x) \subset[a, b]_{K}$ it follows that there is an integer $R$ such that $T^{r}(x) \in[\widehat{a}, b]_{K}$ for all $r \geq R$. By $(\mathrm{AT}), T_{l}\left(T^{r}(x)\right)>0$ for any $r$. We claim that there is a point $y \in \omega(x)$ with $y_{l}>0$. Otherwise, there is 
$\bar{R} \geq R$ such that $\left(T^{r}(x)\right)_{l}<\delta_{0}$ for any $r \geq \bar{R}$. Obviously, $T^{r}(x) \geq_{K} \widehat{a}+\left(T^{r}(x)\right)_{l} e_{l}$. The monotonicity implies that $\left(T^{r+1}(x)\right)_{l} \geq T_{l}\left(\widehat{a}+\left(T^{r}(x)\right)_{l} e_{l}\right) \geq\left(T^{r}(x)\right)_{l}$ for all $r \geq \bar{R}$. Hence $y_{l}>0$ for all $y \in \omega(x)$, a contradiction. The claim holds. Fix $y \in \omega(x)$ with $y_{l}>0$. Then $a \leq_{K} y$, and hence we can choose $0<\delta \ll 1$ such that $\bar{a}=a+\delta e_{l} \leq_{K} y \leq_{K} b$. This shows that $\omega(y)=\omega(\bar{a})=b$, i.e., $b \in \omega(x)$. Since for any $x \in \Gamma$ we have $x<_{K} b$ and $x_{i} \neq b_{i}$ for all $i \in L_{1}$, we can find an integer $r$ such that $x<_{K} T^{r}(x)$, which implies that $\omega(x)$ is a periodic orbit. Together with the fixed point $b \in \omega(x)$, we conclude that $\omega(x)=b$ for all $x \in \Gamma$.

Finally, if $p$ is a fixed point with $a \leq_{K} p$ and $p_{l}>0$, then $\bar{a}=a+\delta e_{l} \leq_{K} p$ for $0<\delta \ll 1$, which implies that $b=\omega(\bar{a}) \leq_{K} p$. The proof is complete.

Lemma 4.4. Suppose that $T: R_{+}^{n} \rightarrow R_{+}^{n}$ satisfies the hypotheses (AT), (BT) and $(\mathrm{CT})$, and that there exists a locally asymptotically stable fixed point $p$ in $R_{+}^{n}$. Then $\omega(x)=p$ for any $x \in \operatorname{Int} R_{+}^{n}$. Furthermore, let $L=\left\{i: p_{i}>0\right\}$. Then the domain of attraction of $p$ is $\Omega=\left\{x \in R_{+}^{n}: x_{i}>0\right.$ for all $\left.i \in L\right\}$.

We shall prove this lemma by induction on the dimension $n$ of $R_{+}^{n}$.

Lemma 4.5. When $n=2$, the conclusion of Lemma 4.4 holds.

Proof. In this case, $N=\{1,2\}, I=\{1\}, J=\{2\}$. The restriction of $T$ to $H_{I}^{+}$or $H_{J}^{+}$is cooperative. By Theorem 3.1, there are $\left(x_{I}^{0}, 0\right) \in H_{I}^{+}$and $\left(0, x_{J}^{0}\right) \in H_{J}^{+}$such that $\omega(x)=\left(x_{I}^{0}, 0\right)$ for all $x \in \operatorname{Int} H_{I}^{+}$and $\omega(x)=\left(0, x_{J}^{0}\right)$ for all $x \in \operatorname{Int} H_{J}^{+}$. For any $x=\left(x_{1}, x_{2}\right) \in \operatorname{Int} R_{+}^{2}$, we have $\left(0, x_{2}\right)<_{K} x<_{K}\left(x_{1}, 0\right)$. Hence $\omega(x) \subset U=$ $\left[\left(0, x_{J}^{0}\right),\left(x_{I}^{0}, 0\right)\right]_{K}$, and $p \in\left[\left(0, x_{J}\right),\left(x_{I}, 0\right)\right]_{K}$.

(1) $p=(0,0)$. Then $p=\left(0, x_{J}^{0}\right)=\left(x_{I}^{0}, 0\right)$, and hence $\omega(x)=p$ for any $x \in R_{+}^{2}$.

(2) $p \in \operatorname{Int} H_{I}^{+}$. Then $p=\left(x_{I}^{0}, 0\right)$. Consider $\Omega_{1}=\left\{x \in R_{+}^{2}, p \leq_{K} x\right\} \subset \operatorname{Int} H_{I}^{+}$. Then $\omega(x)=p$ for every $x \in \Omega_{1}$. Let $\Omega_{2}=\left\{x=\left(x_{1}, x_{2}\right) \in R_{+}^{2}: 0<x_{1} \leq x_{I}^{0}, x_{2}>\right.$ $0\}$. Lemma 4.2 implies that $i(T, U, p)=1$, and $i(T, U, U)=1$. Hence if $q \neq p$ is a fixed point in $U$, then $i(T, U, q)=0$. So there is no other fixed point in $U$ except $p,\left(0, x_{J}^{0}\right),(0,0)$. In fact $x_{J}^{0}$ may be equal to 0 .

By Lemma $4.2, i\left(T, U,\left(0, x_{J}^{0}\right)\right)=0$ implies $\left.\frac{\partial T_{1}}{\partial x_{1}}\right|_{\left(0, x_{J}^{0}\right)}>1$. This condition implies that $T_{1}\left(\left(\delta, x_{J}^{0}\right)\right)>\delta$ for sufficiently small $\delta$. On the other hand, $T\left(\left(\delta, x_{J}^{0}\right)\right)>_{K}$ $T\left(\left(0, x_{J}^{0}\right)\right)=\left(0, x_{J}^{0}\right)$; hence $T\left(\left(\delta, x_{J}^{0}\right)\right)>_{K}\left(\delta, x_{J}^{0}\right)$. So $\omega\left(\left(\delta, x_{J}^{0}\right)\right)=p$; furthermore, by the monotonicity of $T, \omega(x)=p$ for any $x \in U \backslash H_{J}^{+}$. There exists $\bar{x}_{J}^{0}>x_{J}^{0}$ such that $\left.\frac{\partial T_{1}}{\partial x_{1}}\right|_{\left(0, \bar{x}_{J}^{0}\right)}>1$. It follows that $\omega(x) \cap H_{J}^{+}=\emptyset$ for any $x \in \operatorname{Int} R_{+}^{2}$, and we know that $\omega(x) \subset U=\left[\left(0, x_{J}^{0}\right),\left(x_{I}^{0}, 0\right)\right]_{K}$. Hence $\omega(x)=p$.

(3) $p \in \operatorname{Int} H_{J}^{+}$. This case is similar to (2).

(4) $p \in \operatorname{Int} R_{+}^{2}$. In this case, it is easy to see that there exist precisely four fixed points $(0,0),\left(x_{I}^{0}, 0\right),\left(0, x_{J}^{0}\right)$ and $p$ in $R_{+}^{2}$, and $\left(0, x_{J}^{0}\right)<_{K} p,(0,0)<_{K}\left(x_{I}^{0}, 0\right)$. $p \in \operatorname{Int} R_{+}^{2}$ implies $p$ is locally asymptotically stable, and $i(T, U, p)=1$. Hence the other fixed points have index 0 . In the same way as in (2), we can obtain that $\omega(x) \cap H_{I}^{+}=\omega(x) \cap H_{J}^{+}=\emptyset$ for any $x \in \operatorname{Int} R_{+}^{2}$, and $\omega(x)=p$ for any $x \in U \cap \operatorname{Int} R_{+}^{2}$. Hence $\omega(x)=p$ for any $x \in \operatorname{Int} R_{+}^{2}$.

Proof of Lemma 4.4. We shall prove this lemma by induction on the dimension $n$ of $R_{+}^{n}$. The statement is clear if $n=2$ by Lemma 4.5. So assume for induction that the lemma is true when the dimension of the system is less than $n$. Now, we consider an $n$-dimensional system. We divide the proof into three cases.
(1) $p \in H_{I}^{+}$
(2) $p \in H_{J}^{+}$,
(3) $p \notin H_{I}^{+}$and $p \notin H_{J}^{+}$. 
Case (1). Let $p=\left(p_{1}, p_{2}, \cdots, p_{k}, 0,0, \cdots, 0\right)$. Then $p$ is locally asymptotically stable by assumption. In particular, $p$ is locally asymptotically stable with respect to $H_{I}^{+}$. Because of the conclusion of Lemma 3.2, $p$ attracts all points in $\left\{x \in H_{I}^{+}\right.$: $x_{i} \neq 0$ for any $i$ with $\left.p_{i} \neq 0\right\}$. In particular, $p$ attracts all points in $\Omega_{1}=\{x \in$ $\left.R_{+}^{n}, x \geq_{K} p\right\}$. In the following, we consider the set $\Omega_{2}$ defined by

$$
\begin{aligned}
\Omega_{2}=\left\{x \in R_{+}^{n}:\right. & 0<x_{i}<p_{i} \text { for } i \in I \text { with } p_{i}>0 \\
& \left.x_{i^{\prime}}=0 \text { for } i^{\prime} \in I \text { with } p_{i^{\prime}}=0, x_{j}>0 \text { for } j \in J\right\} .
\end{aligned}
$$

If there exists $i \in I$ such that $p_{i}=0$, then $\Omega_{2} \subset H_{N_{i}}^{+}$where $N_{i}=N \backslash\{i\}$, and $p$ is locally asymptotically stable with respect to $H_{N_{i}}^{+}$. Hence, $p$ attracts all the points in $\Omega_{2}$ by the induction assumption. It is not difficult to prove that for any $x \in \Omega$, there exist $y \in \Omega_{2}$ and $z \in \Omega_{1}$ such that $y \leq_{K} x \leq_{K} z$, which implies that $\omega(x)=\omega(y)=\omega(z)=p$. The lemma is true in this situation.

Now, suppose that $p \in \operatorname{Int} H_{I}^{+} . H_{J}^{+}$is an invariant set under $T$, and $T$ is cooperative on $H_{J}^{+}$. Hence there is a fixed point $a=\left(0, \ldots, 0, a_{k+1}, \ldots, a_{n}\right) \in H_{J}^{+}$such that $a$ attracts all points in

$$
\left\{x \in H_{J}^{+}: x_{j}>0 \text { for any } j \in J \text { with } a_{j}>0\right\} .
$$

$U=[a, p]_{K}$ is invariant under $T$. Since $p$ is locally asymptotically stable and $i(T, U, U)=1$, Lemma 4.2 implies that $i(T, U, p)=1$ and $i(T, U, q)=0$ for any other fixed point $q \in U$. Since $i(T, U, a)=0$, by Lemma 4.2 there is $i \in I$ such that $\left.\frac{\partial T_{i}}{\partial x_{i}}\right|_{a}>1$ is an eigenvalue of $D T(a)$. Without loss of generality, let $i=1$. Then $a, L=J$ and $l=1$ satisfy all conditions in Lemma 4.3. Applying it, we have a fixed point $a^{1}$ satisfying (i) through (iii) with $L_{1}=J \cup\{1\}$. In particular, $a^{1} \in[a, p]_{K}$ attracts $\left\{x \in H_{L_{1}}^{+}: 0<x_{1}<a_{1}^{1}, x_{j}>a_{j}^{1}\right.$ for $\left.j \in J\right\}$. By the hyperbolic assumption, $a^{1}$ is locally asymptotically stable in $H_{L_{1}}^{+}$. Therefore if $I \neq\{1\}$, then $a^{1}$ attracts $\operatorname{Int} H_{L_{1}}^{+}$by the induction assumption. Applying Lemma 4.2 again, we get that there is $i \in I \backslash\{1\}$, say $i=2$, such that $\left.\frac{\partial T_{2}}{\partial x_{2}}\right|_{a^{1}}>1$. Again, $a^{1}, L_{1}$ and $l=2$ satisfy all conditions of Lemma 4.3. It follows that there exists a fixed point $a^{2} \in[a, p]_{K}$ such that it attracts the set $\left\{x \in H_{L_{2}}^{+}: 0<x_{i}<a_{i}^{2}\right.$ for $i=1,2, x_{j}>a_{j}^{2}$ for $\left.j \in J\right\}$ with $L_{2}=L_{1} \cup\{2\}$. The hyperbolic assumption implies that $a^{2}$ is locally asymptotically stable in $H_{L_{2}}^{+}$. If $I \neq\{1,2\}$, we conclude that $a^{2}$ attracts $\operatorname{Int} H_{L_{2}}^{+}$by the induction assumption.

After using such a procedure $k$ times, we can find a fixed point $a^{k}=q=$ $\left(q_{1}, q_{2}, \ldots, q_{k}, q_{k+1}, \ldots, q_{n}\right) \in[a, p]_{K}$ with $q_{i}>0$ for $i \in I$. The domain of attraction of $q$ includes

$\Omega_{q}=\left\{x=\left(x_{1}, \ldots, x_{k}, x_{k+1}, \ldots, x_{n}\right): 0<x_{i}<q_{i}\right.$ for $i \in I, x_{j}>q_{j}$ for any $\left.j \in J\right\}$.

Since $q$ is hyperbolic, it is easy to see that $q$ is locally asymptotically stable with respect to $R_{+}^{n}$; in particular, $q$ is locally asymptotically stable with respect to $[a, p]_{K}$. Hence, $q$ is equal to $p$ by the uniqueness of the stable fixed point in $[a, p]_{K}$. Hence, $\Omega_{q}=\Omega_{2}$.

For any $x \in \Omega$, there are $x^{1} \in \Omega_{1}$ and $x^{2} \in \Omega_{2}$ such that $x^{2} \leq_{K} x \leq_{K} x^{1}$. Therefore, $\omega(x)=\omega\left(x^{1}\right)=\omega\left(x^{2}\right)=p$.

Case (2). If Case (1) is true, then pick a transformation by $\left(x_{1}, \ldots, x_{n}\right)$ such that it is changed into Case (1). Actually, we only choose a suitable permutation of species indices for this purpose. 
Case (3). Let $p=\left(p_{1}, \ldots, p_{k}, p_{k+1}, \ldots, p_{n}\right)=\left(p_{I}, p_{J}\right)$. First, consider the set

$$
\begin{aligned}
\Omega_{3}= & \left\{x \in R_{+}^{n}: 0<x_{i}<p_{i} \text { for } i \in I \text { with } p_{i}>0,\right. \\
& \left.x_{i^{\prime}}=0 \text { for } i^{\prime} \in I \text { with } p_{i^{\prime}}=0, x_{j}>p_{j} \text { for } j \in J\right\} .
\end{aligned}
$$

If there is some $i \in I$ such that $p_{i}=0$, then there is some $L \subset N$ such that $\Omega_{3} \subset H_{L}^{+}$. Hence the induction assumption implies $\omega(x)=p$ for any $x \in \Omega_{3}$.

Otherwise, $p_{i}>0$ for any $i \in I$. By Theorem 3.1, there is a fixed point $a \in H_{J}^{+}$ that is globally asymptotically stable with respect to $\operatorname{Int} H_{J}^{+}$. Since $a$ attracts $\operatorname{Int} H_{J}^{+}, a \leq_{K} p$. By Lemma 4.2 and the stability of $p, i\left(T,[a, p]_{K}, p\right)=1$ and the index of every other fixed point in $[a, p]_{K}$ is 0 . Hence there is $i \in I$ such that $\left.\frac{\partial T_{i}}{\partial x_{i}}\right|_{a}>1$ is an eigenvalue of $D T(a)$. Without loss of generality, let $i=1$; then, applying Lemma 4.3 to $a, L=J$ and $l=1$ here, we conclude that there is a fixed point $a^{1} \in[a, p]_{K}$ with the property that it attracts the set $\left\{x \in H_{J \cup\{1\}}^{+}: 0<x_{1}<\right.$ $a_{1}^{1}, x_{j}>a_{j}^{1}$ for any $\left.j \in J\right\}$. Completely repeating the argument in Case (1), we obtain that after $k$ steps we can find $q \in[a, p]_{K}$ that attracts

$$
\begin{aligned}
\Omega_{3}^{q}=\{x & =\left(x_{1}, \ldots, x_{k}, x_{k+1}, \ldots, x_{n}\right): \\
& \left.0<x_{i}<q_{i} \text { for } i \in I, x_{j}>q_{j} \text { for any } j \in J\right\} .
\end{aligned}
$$

This implies that $q$ is locally asymptotically stable with respect to $R_{+}^{n}$ by hyperbolicity. Therefore, $q=p$ by the uniqueness of the stable fixed point in $[a, p]_{K}$; that is, $p$ attracts $\Omega_{3}$.

Similarly, we can obtain that $p$ attracts all points in

$$
\begin{aligned}
\Omega_{4}= & \left\{x \in R_{+}^{n}: x_{i}>p_{i} \text { for } i \in I, 0<x_{j}<p_{j}\right. \text { for } \\
& \left.j \in J \text { with } p_{j}>0, x_{j^{\prime}}=0 \text { for } j^{\prime} \in J \text { with } p_{j^{\prime}}=0\right\} .
\end{aligned}
$$

For any $x \in \Omega$, we can find $x^{3} \in \Omega_{3}$ and $x^{4} \in \Omega_{4}$ such that $x^{3} \leq_{K} x \leq_{K} x^{4}$. Then $\omega(x)=\omega\left(x^{3}\right)=\omega\left(x^{4}\right)=p$. The proof is complete.

Proof of Theorem 4.1. First, Theorem 3.1 implies that there is a fixed point $a=$ $\left(0, \ldots, 0, a_{k+1}, \ldots, a_{n}\right) \in H_{J}^{+}$such that $a$ attracts all points in $\left\{x \in H_{J}^{+}: x_{j}>0\right.$ for any $j \in J$ such that $\left.a_{j}>0\right\}$. If $a$ is locally asymptotically stable with respect to $R_{+}^{n}$, then by Lemma $4.4, p=a$ is the point we want to obtain. Otherwise, there is $i \in I$ such that $\left.\frac{\partial T_{i}}{\partial x_{i}}\right|_{a}>1$ is an eigenvalue of $D T(a)$. Without loss of generality, we may assume $i=1$. Applying Lemma 4.3 to $a, L=J$ and $l=1$ here, we obtain a fixed point $a^{1}>_{K} a$. By (ii) of Lemma 4.3 and Lemma 4.4, $a^{1}$ attracts $\operatorname{Int} H_{L_{1}}^{+}$ with $L_{1}=J \cup\{1\}$. If $a^{1}$ is locally asymptotically stable, then $p=a^{1}$ is the point we want to find. Otherwise we discuss the system in a similar way. At most after the $k$-th step we can find a fixed point $p$ that is locally asymptotically stable. Then Lemma 4.4 implies the domain of attraction of $p$ is $\Omega$. The proof is complete.

Remark 4.6. Similar results for autonomous ordinary differential equations were verified in [7, 15, 16, 17]. Theorem 4.1 unifies quite a number of such individual results.

\section{Convergence FOR Systems With Limited COMPETITION}

In this section, we will provide a result on the convergence of all orbits for dynamical systems with limited competition. 
We say this kind of map $T$ is strong in Int $R_{+}^{n}$ if $T(x) \ll_{K} T(y)$ whenever $x<_{K} y$ and $x, y \in \operatorname{Int} R_{+}^{n}$, where $x \ll_{K} y$ is understood to be $x_{i}<y_{i}$ for any $i \in I$ and $x_{j}>y_{j}$ for any $j \in J$.

Theorem 5.1. Suppose that $T$ is strong in $\operatorname{Int} R_{+}^{n}$ and satisfies the hypotheses (AT) with the following additional conditions:

(i) every boundary fixed point is hyperbolic in $R_{+}^{n}$ and asymptotically stable relative to the face it belongs to;

(ii) every positive fixed point is stable.

Then every orbit converges to a fixed point. Furthermore, if a positive fixed point exists, then the positive fixed point set $F$ is either a singleton or a totally ordered curve such that $\omega(x) \subset F$ for any $x \in \operatorname{Int} R_{+}^{n}$.

Proof. Assume that there is no positive fixed point. Then the conclusion follows immediately from Theorem 4.1. Again applying Theorem 4.1, we know that $\omega(x)$ is a single fixed point if $x \in \partial R_{+}^{n}$. It remains to consider the case that there is a positive fixed point ( $\left.\operatorname{say} p=\left(p_{1}, p_{2}, \cdots, p_{n}\right) \in \operatorname{Int} R_{+}^{n}\right)$ and $x \in \operatorname{Int} R_{+}^{n}$.

Suppose $p_{N_{i}}=p-p_{i} e_{i} \in \operatorname{Int} H_{N_{i}}^{+}$for $1 \leq i \leq k$. Then Theorem 4.1 implies that $\omega\left(p_{N_{i}}\right)=q_{N_{i}} \in H_{N_{i}}^{+}$. Obviously, $p_{N_{i}}<_{K} p$ for any $i \in I$. By monotonicity, $T\left(p_{N_{i}}\right)<_{K} p$. Using (AT), we get that $T\left(p_{N_{i}}\right) \leq_{K} p_{N_{i}}$ for any $i \in I$. Therefore, we have $q_{N_{i}}=\omega\left(p_{N_{i}}\right) \leq_{K} p_{N_{i}}$; in particular, $\left(q_{N_{i}}\right)_{j} \geq\left(p_{N_{i}}\right)_{j}=p_{j}>0$ for all $i \in I$ and $j \in J$. Let

$$
q=\left(q_{1}, \ldots, q_{k}, q_{k+1}, \ldots, q_{n}\right) \text { with } q_{m}=\left\{\begin{array}{cl}
\max _{i \in I}\left(\left(q_{N_{i}}\right)_{m}\right) & \text { if } m \in I \\
\min _{i \in I}\left(\left(q_{N_{i}}\right)_{m}\right) & \text { if } m \in J .
\end{array}\right.
$$

Then $q_{N_{i}} \leq_{K} q \leq_{K} p$ for any $i \in I$. Moreover, $q$ has the property that if $q_{N_{i}} \leq_{K} x$ for any $i \in I$, then $q \leq_{K} x$. The monotonicity implies that $q_{N_{i}}=T\left(q_{N_{i}}\right) \leq K T(q)$ for any $i \in I$; hence $q \leq_{K} T(q)$. In the following we shall consider the order interval $[q, p]_{K}$. We divide the cases into $q \in \operatorname{Int} R_{+}^{n}$ and $q \notin \operatorname{Int} R_{+}^{n}$.

Assume that $q \in \operatorname{Int} R_{+}^{n}$. Then $F \cap[q, p]_{K}$ is either $\{p\}$ or a totally ordered curve by the result of Dancer and Hess in [22], and $\omega(x)$ is a fixed point for any $x \in[q, p]_{K}$. Fix $x \in \operatorname{Int} R_{+}^{n}$ with $x \leq_{K} p$. Then $x_{N_{i}}=x-x_{i} e_{i} \leq_{K} x \leq_{K} p$. Therefore $q_{N_{i}}=\omega\left(x_{N_{i}}\right) \leq_{K} \omega(x) \leq_{K} p$ for each $i \in I$; that is, $\omega(x) \subset[q, p]_{K}$ for any positive point $x \leq_{K} p$. By the invariance and the stability of positive fixed points, $\omega(x)$ is a singleton in $F \cap[q, p]_{K}$.

Assume that $q \notin \operatorname{Int} R_{+}^{n}$. Then there exists an $i \in I$ such that $q \in H_{N_{i}}^{+}$. Since $q_{N_{i}} \leq_{K} q \leq_{K} p$ and $q_{i}=0$, it follows that $q_{N_{i}} \leq_{K} q \leq_{K} p_{N_{i}}$, which implies that $\omega(q)=q_{N_{i}}$. It follows from $q \leq_{K} T(q)$ that $\omega(q)=q_{N_{i}}=q$. It is easy to see that all fixed points in $[q, p]_{K} \backslash\{q\}$ are in Int $R_{+}^{n}$. We claim that $\left.\frac{\partial T_{i}}{\partial x_{i}}\right|_{q}>1$. Otherwise, $q$ is asymptotically stable. Then there is no other fixed point in a small neighborhood of $q$. This implies that there is a minimal element $q^{\prime}$ in the set $F \cap\left([q, p]_{K} \backslash\{q\}\right)$. So the result of Dancer and Hess [22] implies that there is a connecting orbit between $q$ and $q^{\prime}$. However, $q$ and $q^{\prime}$ are stable, a contradiction, which shows that the claim holds.

Using the same method as in the proof of Lemma 4.3, we can easily verify that there is a fixed point $\bar{x} \in \operatorname{Int} R_{+}^{n}$ such that $q \leq_{K} \bar{x} \leq_{K} p$. Furthermore, either $\bar{x}=p$ or $[\bar{x}, p]_{K} \cap F$ is a totally ordered curve and $\omega(x)$ is a singleton in $[\bar{x}, p]_{K}$ for any $x \in \operatorname{Int} R_{+}^{n}$ with $x \leq_{K} p$. Switching the index subsets $I$ and $J$, we can similarly 
prove that $\omega(x)$ is a singleton for any $x \in \operatorname{Int} R_{+}^{n}$ with $p \leq_{K} x$. This completes the proof.

Remark 5.2. Theorem 5.1 is a version of Dancer and Hess [22] and Takáč [29]. However, owing to the invariance of the boundary of $R_{+}^{n}$, there are many unstable fixed points on $\partial R_{+}^{n}$.

\section{Permanence And Coexistence in a positive fixed Point}

In this section, we will study permanence for systems with limited competition and use the result in section 4 to give necessary and sufficient conditions for a system with limited competition to have a globally asymptotically stable positive fixed point.

Let $L$ and $P$ be two subsets of $N, I \subset L, J \subset P, x=\left(x_{I}, x_{J}\right) \in R_{+}^{n}, x_{I} \in$ $R_{+}^{k}, x_{J} \in R_{+}^{n-k}$.

In this section, we always use $u, \bar{u}, u^{0}$, etc. to denote vectors in \#L-dimensional Euclidean space $R^{\# L}$, use $v, \bar{v}, v^{0}$, etc. to denote vectors in $\# \bar{L}$-dimensional Euclidean space $R^{\# \bar{L}}$, use $w, \bar{w}, w^{0}$, etc. to denote vectors in \#P-dimensional Euclidean space $R^{\# P}$, and use $z, \bar{z}, z^{0}$, etc. to denote vectors in $\# \bar{P}$-dimensional Euclidean space $R^{\# \bar{P}}$. Without loss of generality, let $L=\{1,2, \ldots, k, k+1, \ldots, l\}$, $P=\{n-p+1, \ldots, k+1, \ldots, n\}$.

We have the following theorem.

Theorem 6.1. Suppose that $T$ satisfies the hypothesis $(\mathrm{AT}),\left(u^{0}, 0\right)$ and $\left(0, w^{0}\right)$ are two fixed points with $\left(u^{0}, 0\right) \in \operatorname{Int} H_{L}^{+},\left(0, w^{0}\right) \in \operatorname{Int} H_{P}^{+}$and $\left(0, w^{0}\right) \leq_{K}\left(u^{0}, 0\right)$. Suppose that $\left.\frac{\partial T_{i}}{\partial x_{i}}\right|_{\left(u^{0}, 0\right)}>1$ for any $i \in \bar{L}$ and $\left.\frac{\partial T_{j}}{\partial x_{j}}\right|_{\left(0, w^{0}\right)}>1$ for any $j \in \bar{P}$. Then there are positive fixed points $\bar{x}, \widehat{x}$ satisfying the following properties:

(1) $\bar{x}, \widehat{x} \in \operatorname{Int} R_{+}^{n}, \bar{x}, \widehat{x} \in\left[\left(0, w^{0}\right),\left(u^{0}, 0\right)\right]_{K}, \bar{x} \leq_{K} \widehat{x}$.

(2) If $x \in \operatorname{Int} R_{+}^{n},\left(0, w^{0}\right) \leq_{K} x \leq_{K} \bar{x}$, then $\omega(x)=\bar{x}$, and if $x \in \operatorname{Int} R_{+}^{n}, \widehat{x} \leq_{K}$ $x \leq_{K}\left(u^{0}, 0\right)$, then $\omega(x)=\widehat{x}$.

(3) If $x \in \operatorname{Int} R_{+}^{n},\left(0, w^{0}\right) \leq_{K} x \leq_{K}\left(u^{0}, 0\right)$, then $\omega(x) \subset[\bar{x}, \widehat{x}]_{K}$.

In addition, if $\left(u^{0}, 0\right)$ attracts all points $x \in \operatorname{Int} H_{L}^{+}$with $x \geq_{K}\left(u^{0}, 0\right)$, and $\left(0, w^{0}\right)$ attracts all points $x \in \operatorname{Int} H_{P}^{+}$with $x \leq_{K}\left(0, w^{0}\right)$, then $\omega(x)=\widehat{x}$ for all $x \in \operatorname{Int} R_{+}^{n}$ with $x \geq_{K} \widehat{x}, \omega(x)=\bar{x}$ for all $x \in \operatorname{Int} R_{+}^{n}$ with $x \leq_{K} \bar{x}$, and $\omega(x) \subset[\bar{x}, \widehat{x}]_{K}$ for all $x \in \operatorname{Int} R_{+}^{n}$.

Proof. First, we consider the point $\left(0, w^{0}\right)$. In section 2, we saw that $\left.\frac{\partial T_{i}}{\partial x_{j}}\right|_{\left(0, w^{0}\right)}=0$ for any $i \in \bar{P}$ and $j \neq i$. Then there exists a neighborhood $U$ of $\left(0, w^{0}\right)$ such that $\left.\frac{\partial T_{i}}{\partial x_{i}}\right|_{x}>1+n \delta,-\delta<\left.\frac{\partial T_{i}}{\partial x_{j}}\right|_{x}<\delta$ for all $x \in U, i \in \bar{P}$ and $j \neq i$, where $\delta$ is a sufficiently small positive number. Consider $\left(t z, w^{0}\right) \in U$ for $0 \leq t \leq 1$, where $z=\epsilon(1,1, \ldots, 1) \in \operatorname{Int} R_{+}^{\# \bar{P}}$. For any $i \in \bar{P}$,

$$
\begin{aligned}
T_{i}\left(\left(z, w^{0}\right)\right) & =T_{i}\left(\left(z, w^{0}\right)\right)-T_{i}\left(\left(0, w^{0}\right)\right) \\
& =\int_{0}^{1} \operatorname{grad} T_{i}\left(t z, w^{0}\right) d t \bullet(z, 0) \\
& >(1+n \delta) \epsilon-n \delta \epsilon \geq \epsilon .
\end{aligned}
$$

On the other hand, $T\left(\left(z, w^{0}\right)\right) \geq_{K} T\left(\left(0, w^{0}\right)\right)=\left(0, w^{0}\right)$. Therefore, $T\left(\left(z, w^{0}\right)\right) \geq_{K}$ $\left(z, w^{0}\right)$. Hence, there is a fixed point $\bar{x} \geq_{K}\left(z, w^{0}\right)$ such that $\omega\left(\left(z, w^{0}\right)\right)=\bar{x}$. The conclusion holds for all sufficiently small $\epsilon$. The monotonicity of $T$ implies that for 
all $x \in \operatorname{Int} R_{+}^{n}$ we have $\left(0, w^{0}\right) \leq_{K} x \leq_{K} \bar{x}$ and $\omega(x)=\bar{x}$. Similarly, there is a fixed point $\widehat{x} \leq_{K}\left(u^{0}, 0\right)$ which attracts all $x \in \operatorname{Int} R_{+}^{n}$ with $\widehat{x} \leq_{K} x \leq_{K}\left(u^{0}, 0\right)$. Since $\left(0, w^{0}\right) \leq_{K}\left(u^{0}, 0\right)$, it is easy to see that $\bar{x} \leq_{K} \widehat{x}$, and $\bar{x} \geq_{K}\left(z, w^{0}\right), \widehat{x} \leq_{K}\left(u^{0}, v\right)$, where $\left(z, w^{0}\right),\left(u^{0}, v\right) \in \operatorname{Int} R_{+}^{n}$ and $\|z\|,\|v\|$ are sufficiently small. Hence $\bar{x}, \widehat{x} \in$ Int $R_{+}^{n}$. Now suppose that the additional assumptions hold. For all $x \in \operatorname{Int} R_{+}^{n}$ with $x \geq_{K} \widehat{x}$, we can choose $(u, 0) \in \operatorname{Int} H_{L}^{+}$with $\left(u^{0}, 0\right) \leq_{K}(u, 0)$ such that $x \leq_{K}(u, 0)$. Then $\widehat{x} \leq_{K} \omega(x) \leq_{K}\left(u^{0}, 0\right)=\omega((u, 0))$. Because $\left.\frac{\partial T_{i}}{\partial x_{i}}\right|_{\left(u^{0}, 0\right)}>1$ for $i \in \bar{L}$, there is $(\bar{u}, 0)$ with $(\bar{u}, 0)_{m}-\left(u^{0}, 0\right)_{m}>0$ for $m \in I,(\bar{u}, 0)_{j}-\left(u^{0}, 0\right)_{j}<0$ for $j \in L \backslash I$ such that $\left.\frac{\partial T_{i}}{\partial x_{i}}\right|_{(\bar{u}, 0)}>1$ for $i \in \bar{L}$. By the monotonicity, $\left.\frac{\partial T_{i}}{\partial x_{i}}\right|_{(u, 0)} \geq\left.\frac{\partial T_{i}}{\partial x_{i}}\right|_{(\bar{u}, 0)}>1$ for any $(u, 0) \in[\widehat{x},(\bar{u}, 0)]_{K}$. This implies that $\omega(x) \subset \operatorname{Int} R_{+}^{n}$ for $x \in \operatorname{Int} R_{+}^{n}$ with $x \geq_{K} \widehat{x}$. Hence, $\omega(x)=\widehat{x}$. Similarly, $\omega(x)=\bar{x}$ for $x \in \operatorname{Int} R_{+}^{n}$ with $x \leq_{K} \bar{x}$. For any $x \in \operatorname{Int} R_{+}^{n}$, there are $x^{1}, x^{2} \in \operatorname{Int} R_{+}^{n}$ satisfying $x^{1} \leq_{K} \bar{x}, x^{2} \geq_{K} \widehat{x}$ such that $x^{1} \leq_{K} x \leq_{K} x^{2}$. Hence $\omega(x) \subset[\bar{x}, \widehat{x}]_{K}$. The proof is complete.

By this theorem and the conclusions in section 4, we can obtain the theorem for coexistence in a positive fixed point.

Theorem 6.2. Suppose that $T$ satisfies (AT), (BT) and (CT). Then there exists a unique fixed point $p \in \operatorname{Int} R_{+}^{n}$ that is globally asymptotically stable with respect to Int $R_{+}^{n}$ if and only if the following conditions hold.

(1) There exists $L \subset N$ with $I \subset L$ such that there exists a fixed point $\left(u^{0}, 0\right) \in$ Int $H_{L}^{+}$with $\left.\frac{\partial T_{i}}{\partial x_{i}}\right|_{\left(u^{0}, 0\right)}>1$ for any $i \in \bar{L}$.

(2) There exists $P \subset N$ with $J \subset P$ such that there exists a fixed point $\left(0, w^{0}\right) \in$ Int $H_{P}^{+}$with $\left.\frac{\partial T_{j}}{\partial x_{j}}\right|_{\left(0, w^{0}\right)}>1$ for any $j \in \bar{P}$.

Proof. Sufficiency. Suppose that the conditions (1) and (2) hold. By Lemma 4.4, $\left(u^{0}, 0\right)$ and $\left(0, w^{0}\right)$ attract $\operatorname{Int} H_{L}^{+}$and $\operatorname{Int} H_{P}^{+}$respectively. Thus Theorem 6.1 implies that there exists a fixed point $p \in \operatorname{Int} R_{+}^{n}$. By the hypothesis (BT), $p$ is locally asymptotically stable. By Lemma $4.4, p$ is globally asymptotically stable with respect to Int $R_{+}^{n}$.

Necessity. Assume that $p \in \operatorname{Int} R_{+}^{n}$ is globally asymptotically stable with respect to Int $R_{+}^{n}$. By Theorem 3.1, there is a fixed point $a \in \operatorname{Int} H_{J}^{+}$with $a \leq_{K} p$ such that it attracts Int $H_{J}^{+}$. By Lemma 4.2 and the stability of $p, i\left(T,[a, p]_{K}, p\right)=$ 1 and $i\left(T,[a, p]_{K}, q\right)=0$ for any other fixed point $q \in[a, p]_{K}$. In particular, $i\left(T,[a, p]_{K}, a\right)=0$. Lemma 4.2 implies that there is an index $i \in I$ such that $\left.\frac{\partial T_{i}}{\partial x_{i}}\right|_{a}>1$. If $\left.\frac{\partial T_{m}}{\partial x_{m}}\right|_{a}>1$ for any $m \in I$, then we choose $\left(0, w^{0}\right)=a \in \operatorname{Int} H_{J}^{+}$ satisfying (2). Otherwise, applying Lemma 4.3 to $a, L=J$ and $l=i$, we get a fixed point $a^{1} \in \operatorname{Int} H_{L_{1}}^{+}$with $L_{1}=J \cup\{i\}$ such that it attracts $\operatorname{Int} H_{L_{1}}^{+}$. Then $i\left(T,[a, p]_{K}, a^{1}\right)=0$, and there exists $j \in I$ such that $\left.\frac{\partial T_{j}}{\partial x_{j}}\right|_{a^{1}}>1$. If $\left.\frac{\partial T_{r}}{\partial x_{r}}\right|_{a^{1}}>1$ for any $r \in I \backslash\{i\}$, then $\left(0, w^{0}\right)=a^{1}$ satisfies (2). Otherwise, we continue to apply Lemma 4.3 to $a^{1}$. Obviously, after at most $k$ steps, we obtain $P \subset N$ with $J \subset P$ such that there exists a fixed point $\left(0, w^{0}\right) \in \operatorname{Int} H_{P}^{+}$with $\left.\frac{\partial T_{j}}{\partial x_{j}}\right|_{\left(0, w^{0}\right)}>1$ for any $j \in \bar{P}$.

Similarly, there exists $L \subset N$ with $I \subset L$ such that there exists a fixed point $\left(u^{0}, 0\right) \in \operatorname{Int} H_{L}^{+}$satisfying $\left.\frac{\partial T_{i}}{\partial x_{i}}\right|_{\left(u^{0}, 0\right)}>1$ for any $i \in \bar{L}$. The proof is complete.

In nature it is often observed that the struggle for existence between two species competing for the same limited food supply and living space nearly always ends in the complete extinction of one of the species. This phenomenon is known as the 
"principle of competitive exclusion". Mathematically speaking, the species in the subcommunity $L$ will ultimately become extinct if $\left(T^{n}(x)\right)_{i} \rightarrow 0$ as $n \rightarrow+\infty$ for any $i \in L$.

Theorem 6.3. Suppose that $T$ satisfies (AT), (BT) and (CT). Then the subcommunity $L$ will ultimately become extinct if and only if there is a boundary fixed point $p \in \operatorname{Int} H_{\bar{L}}^{+}$with $\bar{L} \neq N$ such that $\left.\frac{\partial T_{i}}{\partial x_{i}}\right|_{p}<1$ for any $i \in L$.

Proof. The sufficiency follows immediately from Lemma 4.4. Suppose that the subcommunity $L$ will ultimately become extinct. Then there cannot exist a positive fixed point. However, Theorem 4.1 tells us that there exists a fixed point $p$ attracting all positive initial points. Therefore, by the definition of extinction, $p$ lies in the boundary of $R_{+}^{n}$, that is, $p \in \operatorname{Int} H_{\bar{L}}^{+}$for some $L \subset N$. By (BT) and (CT), $\left.\frac{\partial T_{i}}{\partial x_{i}}\right|_{p}<1$ for any $i \in L$. The necessity follows.

Before ending this section, we note that Smith [7] studied permanence for autonomous ordinary differential equations (1.1) and obtained a permanent result in the case $L=I$ and $P=J$. Compared to his, the systems we have considered are more general and the conditions presented are much weaker.

\section{Applications}

Consider the system of ordinary differential equations (1.1) in which $x_{i}$ represents the population density of the $i$-th species and $f_{j}(x, t)$ represents the per capita growth rate of the $j$-th species. Assume that $f_{j}(x, t)$ is continuously differentiable in $R_{+}^{n} \times R$ for every $j \in N$.

The system (1.1) is called cooperative if, for every $(x, t) \in R_{+}^{n} \times R$,

$$
\frac{\partial f_{i}}{\partial x_{j}}(x, t) \geq 0, \quad i \neq j
$$

and we say (1.1) is a system with limited competition if for every $(x, t) \in R_{+}^{n} \times R$,

$$
\left\{\begin{array}{l}
\frac{\partial f_{i}}{\partial x_{j}}(x, t) \geq 0, \text { for } i, j \in I \text { or } i, j \in J, \text { and } i \neq j, \\
\frac{\partial f_{i}}{\partial x_{j}}(x, t) \leq 0, \text { for } i \in I \text { and } j \in J \text { or } i \in J \text { and } j \in I,
\end{array}\right.
$$

where $N=I \cup J$ and $I=\{1,2, \ldots, k\}, J=\{k+1, \ldots, n\}$.

Now, we can conclude the following theorem.

Theorem 7.1. Let $f(x, t)=\left(f_{1}(x, t), \ldots, f_{n}(x, t)\right)$ be continuously differentiable

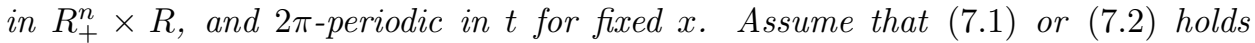
and that every solution with $x\left(t_{0}\right) \geq 0$ of $(1.1)$ can be continued to $\left[t_{0},+\infty\right)$ and is bounded in $R_{+}^{n}$. If every $2 \pi$-periodic solution of (1.1) is hyperbolic (that is, its Floquet multipliers do not lie on the unit circle in the complex plane) and is asymptotically stable with respect to the face it belongs to, then (1.1) has a $2 \pi$ periodic solution $\varphi(t)$ which attracts all solutions whose initial points are in $\{x \geq$ $0: x_{i}>0$ for $i \in N$ with $\left.\varphi_{i}(0)>0\right\}$. Furthermore, for a cooperative system (1.1), there exists a positive $2 \pi$-periodic solution that is globally asymptotically stable in Int $R_{+}^{n}$ if and only if there exists a $2 \pi$-periodic solution $\varphi(t) \in \operatorname{Int} H_{L}^{+}$with $L \subset N$ such that all those Floquet multipliers corresponding to indices $j \in \bar{L}$ are larger than 1. Suppose that the system (1.1) has limited competition. Then (1.1) has a globally asymptotically stable positive $2 \pi$-periodic solution in $\operatorname{Int} R_{+}^{n}$ if and only if 
(1) there is $L \subset N$ with $I \subset L$ such that (1.1) has a $2 \pi$-periodic solution in Int $H_{L}^{+}$and all those Floquet multipliers corresponding to indices $j \in \bar{L}$ are larger than 1; and

(2) there is $P \subset N$ with $J \subset P$ such that (1.1) has a $2 \pi$-periodic solution in Int $H_{P}^{+}$and all those Floquet multipliers corresponding to indices $i \in \bar{P}$ are larger than 1 .

Corollary 7.2. Let $f$ be independent of $t$. Assume that all solutions of (1.1) are bounded in $R_{+}^{n}$. If every equilibrium is hyperbolic and asymptotically stable with respect to the face it belongs to, then there exists an equilibrium $p$ which attracts all points in $\left\{x \geq 0: x_{i}>0\right.$ if $\left.p_{i}>0\right\}$. Furthermore, if (1.1) is an autonomous cooperative system, then it has a positive equilibrium $p$ such that it is globally asymptotically stable if and only if there exists a boundary equilibrium $q \in \operatorname{Int} H_{L}^{+}$such that $f_{i}(q)>0$ for $i \in \bar{L}$. If (1.1) is a system with limited competition, then it has a positive equilibrium that is globally asymptotically stable in $\operatorname{Int} R_{+}^{n}$ if and only if

(1) there exists an equilibrium $p \in \operatorname{Int} H_{L}^{+}$, where $L \subset N$ with $I \subset L$, such that $f_{i}(p)>0$ for $i \in \bar{L}$; and

(2) there exists an equilibrium $q \in \operatorname{Int} H_{P}^{+}$, where $P \subset N$ with $J \subset P$, such that $f_{i}(q)>0$ for $i \in \bar{P}$.

We remark that Corollary 7.2 covers a number of results in [7, 15, 16, 17, 25, 26], but the conditions are a little weaker and the result is more general.

The asymptotic behavior of solutions of the following classical Lotka-Volterra competition model with diffusion has been studied extensively:

$$
\left\{\begin{array}{l}
\frac{\partial u_{1}}{\partial t}=d_{1} \Delta u_{1}+u_{1}\left(r_{1}-a_{11} u_{1}-a_{12} u_{2}\right) \text { in } \Omega_{T} \\
\frac{\partial u_{2}}{\partial t}=d_{2} \Delta u_{2}+u_{2}\left(r_{2}-a_{21} u_{1}-a_{22} u_{2}\right) \text { in } \Omega_{T} \\
\frac{\partial u_{1}}{\partial \nu}=\frac{\partial u_{2}}{\partial \nu}=0 \text { on } \partial \Omega_{T} \\
u_{1}(x, 0)=u_{10}(x), u_{2}(x, 0)=u_{20}(x) \text { in } \Omega
\end{array}\right.
$$

where $\Omega$ is a bounded smooth domain of $R^{N}$ with $N \geq 1, \partial \Omega$ and $\bar{\Omega}$ are the boundary and the closure of $\Omega$, respectively, $\Omega_{T}=\Omega \times[0, T)$ and $\partial \Omega_{T}=\partial \Omega \times[0, T)$ for some $T \in(0, \infty], \nu$ is the outward unit normal vector on $\partial \Omega, r_{i}$ and $a_{i j}(i, j=$ $1,2)$ are all positive constants. The initial values $u_{10}$ and $u_{20}$ are non-negative continuous functions that are not identically zero.

The best results for (7.3) are summarized as follows (see [19], 20], 21]):

(I) when $r_{1} / r_{2}>\max \left(a_{11} / a_{21}, a_{12} / a_{22}\right)$, every solution of $(7.3)\left(u_{1}, u_{2}\right) \rightarrow$ $\left(r_{1} / a_{11}, 0\right)$ uniformly as $t \rightarrow \infty$;

(II) when $r_{1} / r_{2}<\min \left(a_{11} / a_{21}, a_{12} / a_{22}\right),\left(u_{1}, u_{2}\right) \rightarrow\left(0, r_{2} / a_{22}\right)$ uniformly as $t \rightarrow \infty$

(III) when $a_{11} / a_{21}>r_{1} / r_{2}>a_{12} / a_{22},\left(u_{1}, u_{2}\right) \rightarrow u^{*}$ uniformly as $t \rightarrow \infty$, where $u^{*}$ is the solution of the equations $a_{11} u_{1}+a_{12} u_{2}=r_{1}, a_{21} u_{1}+a_{22} u_{2}=r_{2}$.

If the number of species is greater than 2, the asymptotic behavior for such systems is not clear so far. Applying our results above, we can give a perfect generalization for the results on two species stated above. 
Now we consider

$$
\left\{\begin{array}{l}
\frac{\partial u}{\partial t}=\operatorname{diag}\left(d_{1}, \ldots, d_{n}\right) \triangle u+\operatorname{diag}(u)(r+M u) \text { in } \Omega_{T} \\
\frac{\partial u}{\partial \nu}=0 \text { on } \partial \Omega_{T} \\
u(x, 0)=u^{0}(x) \text { in } \Omega .
\end{array}\right.
$$

We assume that (7.4) satisfies the Standard Assumptions:

$\Omega$ is a bounded smooth domain of $R^{N}$ with $N \geq 1, \partial \Omega$ and $\bar{\Omega}$ are the boundary and the closure of $\Omega$, respectively, $\Omega_{T}=\Omega \times[0, T)$ and $\partial \Omega_{T}=\partial \Omega \times[0, T)$ for some $T \in(0, \infty], \nu$ is the outward unit normal vector on $\partial \Omega, d_{i}>0$ for $1 \leq i \leq n$, $u(x, t)=\left(u_{1}(x, t), \ldots, u_{n}(x, t)\right)$, every component of the initial value $u^{0}$ is a nonnegative continuous function, $r=\left(r_{1}, \ldots, r_{n}\right)$ with every $r_{i} \neq 0$, and the constant matrix $M$ has the form (1.2).

The asymptotic behavior of solutions for (7.4) is closely related to that of solutions of the Lotka-Volterra system of ordinary differential equations

$$
\frac{d u}{d t}=\operatorname{diag}(u)(r+M u)
$$

For example, in any case of (I), (II) and (III), the equilibria of (7.5), which is a two-dimensional system, are hyperbolic and locally asymptotically stable relative to the faces they belong to. Now we can generalize it to the following.

Corollary 7.3. Assume that (7.4) satisfies the Standard Assumptions. If every equilibrium of (7.5) is hyperbolic and asymptotically stable relative to the face it belongs to, then there is a constant steady state $u^{*}$ which attracts every solution of (7.4) with initial value $u^{0}(x)$ satisfying that there exists $x \in \Omega$ such that $u_{i}^{0}(x)>0$ for each $i$ with $u_{i}^{*}>0$.

Moreover, there exists a positive steady state $u^{*}$ which attracts all initial values $u^{0}(x)$ with the property that for each $i$, there exists $x \in \Omega$ such that $u_{i}^{0}(x)>0$ if and only if the conditions (1) and (2) in Corollary 7.2 hold, where $f(u)=r+M u$.

Proof. Since we consider the asymptotic behavior of the system (7.4), $T$ is surely $\infty$. Theorem 7.3.1 in Smith [27] guarantees the existence, uniqueness and smoothness of the solution of (7.4). We also know if $u(x, t)$ is a solution of $(7.4)$, then $u(x, t) \in R_{+}^{n}$ for any $(x, t) \in \bar{\Omega} \times[0, T)$.

By the result of Conway [28], if $u(x, t)$ is the solution of (7.4) and $u_{i}^{0}(x)>0$ for some $x \in \Omega$, then $u_{i}(x, t)>0$ for $(x, t) \in \bar{\Omega} \times(0, T)$. Therefore, without loss of generality, we may assume that either $u_{i}^{0}(x) \equiv 0$ on $\bar{\Omega}$ or $u_{i}^{0}(x)>0$ on $\bar{\Omega}$ for each $i$, and furthermore, in order to prove this corollary, we can also assume that $u_{i}^{0}(x)>0$ on $\bar{\Omega}$ for all $i$. Let $a=\left(a_{1}, \ldots, a_{n}\right), b=\left(b_{1}, \ldots, b_{n}\right)$ be defined by

$$
\begin{aligned}
& a_{i}=\min _{x \in \bar{\Omega}} u_{i}^{0}(x) \text { and } b_{i}=\max _{x \in \bar{\Omega}} u_{i}^{0}(x) \text { for } i \in I, \\
& a_{j}=\max _{x \in \bar{\Omega}} u_{j}^{0}(x) \text { and } b_{j}=\min _{x \in \bar{\Omega}} u_{j}^{0}(x) \text { for } j \in J .
\end{aligned}
$$

Then $a, b \in \operatorname{Int} R_{+}^{n}, a \leq_{K} u^{0}(x) \leq_{K} b$. By the maximum principle, we can prove that

$$
\varphi_{t}(a) \leq_{K} u(x, t) \leq_{K} \varphi_{t}(b) \text { for all } t>0,
$$

where $\varphi_{t}(u)$ denotes the solution flow of (7.5), which is obviously the solution of (7.4) with initial value $u$. The detailed proof can be seen in Theorem 7.3.4 of [27], 
where the order is generated by the cone $R_{+}^{n}$. The proof is quite the same if the cone $R_{+}^{n}$ is replaced by $K$. By assumption, there exists a constant steady state $u^{*}$ such that $\lim _{t \rightarrow \infty} \varphi_{t}(a)=\lim _{t \rightarrow \infty} \varphi_{t}(b)=u^{*}$. This implies that $\lim _{t \rightarrow \infty} u(x, t)=u^{*}$. Thus the conclusions of Corollary 7.3 hold.

\section{ACKNOWLEDGEMENTS}

The authors are greatly indebted to the anonymous referee and Prof. Bates for their valuable suggestions leading to an improvement of the manuscript. The second author is also grateful to Prof. Smith for sending a preprint of his paper 4].

\section{REFERENCES}

1. P. Hess and A. C. Lazer, On an abstract competition model and applications, Nonlinear Analysis 16(1991), pp. 917-940. MR 92f:92036

2. S. B. Hsu, H. L. Smith and P. Waltman, Competitive exclusion and coexistence for competitive systems on ordered Banach spaces, Trans. Amer. Math. Soc., 348(1996), pp. 4083-4094. MR 97d:92021

3. S. B. Hsu, P. Waltman and S. Ellermeyer, A remark on the global asymptotic stability of a dynamical system modelling two species in competition, Hiroshima J. Math., 24(1994), pp. 435-446. MR 95h:35115

4. H. L. Smith and H. R. Thieme, Stable coexistence and bi-stability for competitive systems on ordered Banach spaces, J. Diff. Equations 176(2001), 195-222. CMP 1861187

5. Y. Takeuchi, N. Adachi and H. Tokumaru, Global stability of ecosystems of the generalized Volterra type, Math. Biosci., 42(1978), pp. 119-136. MR 80i:92019]

6. C. C. Travis and W. M. Post III, Dynamics and comparative statistics of mutualistic communities, J. Theor. Biol., 78(1979), pp. 553-571. MR 80d:92035

7. H. L. Smith, Competing subcommunities of mutualists and a generalized Kamke theorem, SIAM J. Appl. Math., 46(1986), pp. 856-874. MR 87i:92047

8. H. L. Smith, Systems of ordinary differential equations which generate an order preserving flow. A survey of results, SIAM Review., 30(1988), pp. 87-113. MR 89f:34065

9. M. Hirsch, Systems of differential equations which are competitive or cooperative I: Limit sets, SIAM J. Math. Anal., 13(1982), pp. 167-179. MR 83i:58081

10. M. Hirsch, Systems of differential equations which are competitive or cooperative II: Convergence almost everywhere, SIAM J. Math. Anal., 16(1985), pp. 423-439. MR 87a:58137

11. M. Hirsch, Stability and convergence in strongly monotone dynamical systems, J. reine angew. Math., 383(1988), pp. 1-53. MR 89c:58108

12. H. I. Freedman and P. Waltman, Persistence in models of three interacting predator-prey populations, Math. Biosci., 68(1984), pp. 213-231. MR 85h:92037

13. G. Butler and P. Waltman, Persistence in dynamical systems, J. Differential Equations 63(1986), pp. 255-263. MR 87k:54058

14. G. Butler, H. I. Freedman and P. Waltman, Uniformly persistent systems, Proc. Amer. Math. Soc., 96(1986) 425-430. MR 87d:58119

15. Y. Takeuchi and N. Adachi, The existence of globally stable equilibria of ecosystems of the generalized Volterra type, J. Math. Biol., 10(1980), pp. 401-415. MR 82e:92048

16. C. F. Tu and J. F. Jiang, Global stability and permanence for a class of type $K$ monotone systems, SIAM J. Math. Anal., 30(1999), pp. 360-378. MR 99j:34073

17. C. F. Tu and J. F. Jiang, The necessary and sufficient conditions for the global stability of type-K Lotka-Volterra systems, Proc. Amer. Math. Soc., 127(1999), pp. 3181-3186. MR 2000b:34082

18. I. Tereščák, Dynamics of $C^{1}$ smooth strongly monotone discrete-time dynamical systems, preprint.

19. Y. Lou and W. M. Ni, Diffusion, self-diffusion and cross-diffusion, J. Differential Equations, 131(1996), pp. 79-131. MR 97i:35086

20. P. N. Brown, Decay to uniform states in ecological interactions, SIAM J Appl. Math., 38(1980), pp. 22-37. MR 81a:35047 
21. P. de Mottoni, Qualitative analysis for some quasilinear parabolic systems, Inst. Math. Pol. Acad. Sci., zam., 11/79 190(1979).

22. E. Dancer and P. Hess, Stability of fixed points for order-preserving discrete-time dynamical systems, J. reine angew. Math., 419(1991), pp. 125-139. MR 92i:47088

23. J. F. Jiang, On the global stability of cooperative systems, Bull. London Math. Soc. , 26(1994), pp. 455-458. MR 95i:34089

24. H. Amann, Fixed point equations and nonlinear eigenvalue problems in ordered Banach spaces, SIAM Review., 18(1976), pp. 620-709. MR 54:3519

25. H. L. Smith, On the asymptotic behavior of a class of deterministic models of cooperative species, SIAM J. Appl. Math., 46(1986), pp. 368-375. MR 87j:34066

26. B. S. Goh, Stability in models of mutualism, American Naturalist, 113(1979), pp. 261-275. MR 82h:92045

27. H. L. Smith, Monotone Dynamical Systems: An Introduction to the Theory of Competitive and Cooperative Systems, Math. Surveys Monographs 41, American Mathematical Society, Providence, RI, 1995. MR 96c:34002

28. E. D. Conway, A comparison technique for systems of reaction-diffusion equations, Comm. In Partial Differential Equations, 2(7)(1977), pp. 679-697. MR 56:3436

29. P. Takáč, Convergence to equilibrium on invariant d-hypersurfaes for strongly increasing discrete-time semigroups, J. Math. Anal. Appl., 148(1990), pp. 223-244. MR 91d:58125

Department of Mathematics University of Science and Technology of China Hefei, Anhui 230026, P. R. China

E-mail address: xliang@mail.ustc.edu.cn

Department of Mathematics University of Science and Technology of China Hefei, Anhui 230026, P. R. China

E-mail address: jiangjf@ustc.edu.cn 